\title{
Function theory in sectors
}

\author{
by \\ Brian Jefferies (Sydney)
}

\begin{abstract}
It is shown that there is a one-to-one correspondence between uniformly bounded holomorphic functions of $n$ complex variables in sectors of $\mathbb{C}^{n}$, and uniformly bounded functions of $n+1$ real variables in sectors of $\mathbb{R}^{n+1}$ that are monogenic functions in the sense of Clifford analysis. The result is applied to the construction of functional calculi for $n$ commuting operators, including the example of differentiation operators on a Lipschitz surface in $\mathbb{R}^{n+1}$.
\end{abstract}

1. Introduction. The ability to form functions of continuous linear operators is essential to many applications of functional analysis. In quantum mechanics, the projection $\chi_{E}(A)$ associated with a selfadjoint operator $A$ and the characteristic function $\chi_{E}$ of a Borel subset $E$ of the real line represents the observation that the observable associated with $A$ has values in the set $E$. For general linear operators, there is not such an extensive functional calculus as there is for selfadjoint operators.

Given a bounded linear operator $A$ acting on a Banach space $X$, the Riesz-Dunford functional calculus associates the bounded linear operator $f(A)$ with $A$ by means of the formula

$$
f(A)=\frac{1}{2 \pi i} \int_{C}(\lambda I-A)^{-1} f(\lambda) d \lambda .
$$

Here $f$ is holomorphic in a neighbourhood $U$ of the spectrum $\sigma(A)$ of $A$ in $\mathbb{C}$ and $C$ is a simple closed curve about $\sigma(A)$ and contained in the domain $U$ of $f$.

A higher-dimensional analogue of the Riesz-Dunford functional calculus has recently been investigated using the techniques of Clifford analysis [5]. The analogue of equation (1) for functions of $n$ bounded linear operators

2000 Mathematics Subject Classification: Primary 47A60, 46H30; Secondary 47A25, $30 \mathrm{G} 35$.

Key words and phrases: Clifford algebra, monogenic function, holomorphic function, functional calculus. 
$A_{1}, \ldots, A_{n} \in \mathcal{L}(X)$ is

$$
f\left(A_{1}, \ldots, A_{n}\right)=\int_{\partial \Omega} G_{x}\left(A_{1}, \ldots, A_{n}\right) \boldsymbol{n}(x) f(x) d \mu(x),
$$

for a suitable bounded open subset $\Omega$ of $\mathbb{R}^{n+1}$ with smooth oriented boundary $\partial \Omega$, outward unit normal $\boldsymbol{n}(x)$ at $x \in \partial \Omega$ and surface measure $\mu$. The function $f$ is assumed to be left monogenic in a neighbourhood of $\bar{\Omega}$ in $\mathbb{R}^{n+1}$, that is, it takes values in a Clifford algebra $C \ell\left(\mathbb{C}^{n}\right)$ and satisfies higher-dimensional analogues of the Cauchy-Riemann equations.

Although the simplicity of formula (2) is appealing, the main difficulty is making sense of the Cauchy kernel $x \mapsto G_{x}\left(A_{1}, \ldots, A_{n}\right)$ and determining its set $\gamma\left(A_{1}, \ldots, A_{n}\right)$ of singularities, called the monogenic spectrum of the $n$-tuple $A=\left(A_{1}, \ldots, A_{n}\right)$. Under the assumption that the spectrum of each operator $\sum_{j=1}^{n} \xi_{j} A_{j}$ is real for $\xi \in \mathbb{R}^{n}$, it turns out that $\gamma(A)$ is a nonempty compact subset of $\mathbb{R}^{n}$ and formula (2) defines a function $f(A) \in \mathcal{L}(X)$ of the $n$-tuple $A$ for any function $f$ which is real-analytic in a neighbourhood of $\gamma(A)$ in $\mathbb{R}^{n}[5$, Theorem 3.5]. Such a function automatically has a monogenic extension to a neighbourhood of $\gamma(A)$ in $\mathbb{R}^{n+1}$.

These ideas make sense even if the operators $A_{1}, \ldots, A_{n}$ do not commute with one another. If the $n$-tuple $A$ has a Weyl functional calculus [1], that is, it satisfies suitable exponential growth conditions, then formula (2) is the restriction to real-analytic functions $f$ of the Weyl functional calculus $f \mapsto f(A)$ [4, Corollary 5.5].

The approach to joint functional calculi just mentioned was motivated by A. McIntosh's study of the commuting $n$-tuple $D_{\Sigma}=\left(D_{1}, \ldots, D_{n}\right)$ of differentiation operators on a Lipschitz surface $\Sigma$ in $\mathbb{R}^{n+1}$ in relation to irregular boundary value problems (see [10]). In the case that $\Sigma$ is just the flat surface $\mathbb{R}^{n}$, the operators

$$
D_{j}=\frac{1}{i} \frac{\partial}{\partial x_{j}}, \quad j=1, \ldots, n,
$$

commute with each other and are selfadjoint, otherwise, the unbounded operators $D_{j}, j=1, \ldots, n$, have spectra $\sigma\left(D_{j}\right)$ contained in a complex sector $S_{\omega}(\mathbb{C})=\{ \pm z:|\arg (z)| \leq \omega\}$ with an angle $\omega$ depending on the variation of the directions normal to the surface $\Sigma$.

Arguing by analogy with the situation where $\Sigma=\mathbb{R}^{n}$, functions $\widetilde{f}\left(D_{1}, \ldots, D_{n}\right)$ of the $n$-tuple $D_{\Sigma}$ may be thought of as Fourier multiplier operators acting on $L^{p}(\Sigma)$ for $1<p<\infty$, with the multiplier $\widetilde{f}$ being a bounded holomorphic function defined on some sector in $\mathbb{C}^{n}[10$, Section 5.6.1]. On the other hand, we can use formula (2) to define $f\left(D_{1}, \ldots, D_{n}\right)$ if $f$ is a left monogenic function with suitable decay in a sector in $\mathbb{R}^{n+1}$. This suggests a connection between left monogenic functions defined on a sector 
in $\mathbb{R}^{n+1}$ and bounded holomorphic functions defined on a sector in $\mathbb{C}^{n}$. This paper is devoted to showing that the mapping $f \mapsto \widetilde{f}$ is a bijection from left monogenic functions, uniformly bounded on subsectors of a fixed sector in $\mathbb{R}^{n+1}$, onto the space of holomorphic functions, uniformly bounded on subsectors of a corresponding sector in $\mathbb{C}^{n}$. The holomorphic function $\tilde{f}$ is simply the analytic continuation to a suitable sector in $\mathbb{C}^{n}$ of $f$ restricted to $\mathbb{R}^{n} \backslash\{0\}$.

Given the bounded left monogenic function $f$, the corresponding holomorphic function $\widetilde{f}$ is constructed by applying the Cauchy integral formula (2) to the commuting $n$-tuple $\zeta=\left(\zeta_{1}, \ldots, \zeta_{n}\right) \in \mathbb{C}^{n}$ of multiplication operators in the Clifford algebra $C \ell\left(\mathbb{C}^{n}\right)$, that is,

$$
\widetilde{f}(\zeta)=\int_{\partial \Omega} G_{x}(\zeta) \boldsymbol{n}(x) f(x) d \mu(x) .
$$

Then $\tilde{f}(x)=f(x)$ for all nonzero $x \in \mathbb{R}^{n}$ by the Cauchy integral formula of Clifford analysis. Care needs to be exercised in forming the Cauchy kernel $x \mapsto G_{x}(\zeta)$ for $\zeta \in \mathbb{C}^{n}$. Its set of singularities $\gamma(\zeta) \subset \mathbb{R}^{n+1}$ is the monogenic spectrum of the complex vector $\zeta$, viewed as an $n$-tuple of multiplication operators in $C \ell\left(\mathbb{C}^{n}\right)$. Because the Cauchy kernel $G_{x}(\zeta)$ has the right scaling properties, a uniform bound for $f$ on a sector in $\mathbb{R}^{n+1}$ gives a uniform bound for $\tilde{f}$ on a sector in $\mathbb{C}^{n}$. In the other direction, reconstructing $f$ from $\widetilde{f}$, so showing that the mapping $f \mapsto \widetilde{f}$ is onto, requires the Fourier theory developed by Li, McIntosh and Qian in [8].

Because we can simply multiply two bounded holomorphic functions $\widetilde{f}, \widetilde{g}$ to obtain another $\widetilde{f} \cdot \widetilde{g}$, we also show that there is a Cauchy-Kowalewski product $(f, g) \mapsto f \cdot \ell g$ for all bounded left monogenic functions defined in a sector $S$ in $\mathbb{R}^{n+1}$. A standard series expansion gives a left monogenic function $f \cdot \ell g$ defined in a neighbourhood of $\mathbb{R}^{n} \backslash\{0\}$ coinciding with the product function $f g$ on $\mathbb{R}^{n} \backslash\{0\}$. It is not so obvious that $f \cdot \ell g$ actually extends left monogenically to a bounded function defined on all of $S$.

Both Clifford analysis and the theory of functions of several complex variables are extensions of classical complex analysis. The Cauchy integral formula of Clifford analysis is especially well adapted to operator theory. The main result of the present work suggests that we can pass sometimes from one to the other point of view in some applications. The situation with domains other than sectors and other function spaces remains to be investigated.

We start with a brief résumé of Clifford analysis [2,3] and the monogenic functional calculus treated in [5]. The correspondence $f \mapsto \widetilde{f}$ arises by considering the spectral theory of the commuting $n$-tuple $\left(\zeta_{1}, \ldots, \zeta_{n}\right) \in \mathbb{C}^{n}$ of multiplication operators in the Clifford algebra and this is examined in Section 2, where the main result, Theorem 2.4, and its corollary concerning 
the Cauchy-Kowalewski product are stated. In Section 3, a scaling argument is used to show that the mapping $f \mapsto \widetilde{f}$ introduced in Section 2 actually sends bounded left monogenic functions to bounded holomorphic functions on sectors. That the mapping is onto is proved in Section 4 using Fourier analysis, so completing the proof of Theorem 2.4. Section 5 is devoted to the construction of an $H^{\infty}$-functional calculus for a system of operators of type $\omega$ using the Cauchy integral formula (2).

1.1. The Clifford algebra $C \ell\left(\mathbb{C}^{n}\right)$. The real and imaginary parts of $z \in$ $\mathbb{C}$ are denoted by $\Re(z)$ and $\Im(z)$ respectively, and for an element $\zeta=$ $\left(\zeta_{1}, \ldots, \zeta_{n}\right)$ of $\mathbb{C}^{n}$, the vector $\Re(\zeta)=\left(\Re\left(\zeta_{1}\right), \ldots, \Re\left(\zeta_{n}\right)\right) \in \mathbb{R}^{n}$ denotes the real part of $\zeta$ and $\Im(\zeta)=\left(\Im\left(\zeta_{1}\right), \ldots, \Im\left(\zeta_{n}\right)\right) \in \mathbb{R}^{n}$ denotes the imaginary part of $\zeta$.

Let $C \ell\left(\mathbb{C}^{n}\right)$ be the Clifford algebra generated over the field $\mathbb{C}$ by the standard basis vectors $e_{0}, e_{1}, \ldots, e_{n}$ of $\mathbb{C}^{n+1}$ with conjugation $u \mapsto \bar{u}$. Then $e_{0}$ is the unit of $C \ell\left(\mathbb{C}^{n}\right), e_{j}$ and $e_{k}$ anticommute for $j, k=1, \ldots, n$ and $j \neq k$, and $e_{j}^{2}=-1$ for $j=1, \ldots, n$. The conjugation satisfies $\bar{e}_{0}=e_{0}, \bar{e}_{j}=-e_{j}$ for $j=1, \ldots, n$ and $\overline{u v}=\bar{v} \bar{u}$ for all $u, v \in C \ell\left(\mathbb{C}^{n}\right)$.

The Clifford algebra $C \ell\left(\mathbb{C}^{n}\right)$ is a complex vector space with a basis $e_{S}$, $S \subset\{1, \ldots, n\}$, given by $e_{S}=e_{j_{1}} \cdots e_{j_{k}}$ if $S=\left\{j_{1}, \ldots, j_{k}\right\}$ and $1 \leq j_{1}<$ $\cdots<j_{k} \leq n$ is an ordered enumeration of $S$. If $S=\emptyset$, then $e_{\emptyset}=e_{0}$. In particular, $C \ell\left(\mathbb{C}^{n}\right)$ has complex dimension $2^{n}$. A function $f: U \rightarrow C \ell\left(\mathbb{C}^{n}\right)$ therefore has a unique representation $f=\sum_{S} f_{S} e_{S}$ in which $f_{S}: U \rightarrow \mathbb{C}$ are scalar-valued functions for each subset $S$ of $\{1, \ldots, n\}$.

The embedding $z \mapsto z e_{0}, z \in \mathbb{C}$, identifies $\mathbb{C}$ with a closed commutative subalgebra of $C \ell\left(\mathbb{C}^{n}\right)$ and $\mathbb{C}^{n+1}$ is identified with the closed linear subspace of all elements $z_{0} e_{0}+z_{1} e_{1}+\cdots+z_{n} e_{n}$ of $C \ell\left(\mathbb{C}^{n}\right)$ with $z_{j} \in \mathbb{C}, j=0,1, \ldots, n$. Then $\mathbb{C}^{n}$ is always identified with the subspace $\{0\} \times \mathbb{C}^{n}$ of $\mathbb{C}^{n+1}$ and then with the corresponding subspace of $C \ell\left(\mathbb{C}^{n}\right)$. Similarly, $\mathbb{R}, \mathbb{R}^{n}$ and $\mathbb{R}^{n+1}$ are identified with the corresponding real linear subspaces of $C \ell\left(\mathbb{C}^{n}\right)$.

1.2. Clifford analysis. The generalised Cauchy-Riemann operator is given by

$$
D=\sum_{j=0}^{n} e_{j} \frac{\partial}{\partial x_{j}}
$$

Let $U \subset \mathbb{R}^{n+1}$ be an open set. A function $f: U \rightarrow C \ell\left(\mathbb{C}^{n}\right)$ is called left monogenic if $D f=0$ in $U$ and right monogenic if $f D=0$ in $U$.

REMARK 1.1. Each component $f_{S}$ of a left monogenic function $f=$ $\sum_{S} f_{S} e_{S}$ is harmonic in $\mathbb{R}^{n+1}$, because if

$$
\bar{D}=\sum_{j=0}^{n} \bar{e}_{j} \frac{\partial}{\partial x_{j}}=e_{0} \frac{\partial}{\partial x_{0}}-e_{1} \frac{\partial}{\partial x_{1}}-\cdots-e_{n} \frac{\partial}{\partial x_{n}}
$$


denotes the operator conjugate to $D$, then we have

$$
\sum_{S}\left(\Delta f_{S}\right) e_{S}=\Delta f=\left(\Delta e_{0}\right) f=(\bar{D} D) f=\bar{D}(D f)=0 .
$$

Hence, $\Delta f_{S}=0$ for all $S \subset\{1, \ldots, n\}$. The same remark applies to right monogenic functions.

REMARK 1.2. In the simplest nontrivial case of $n=2$, each function with values in $C \ell\left(\mathbb{C}^{2}\right)$ can be written as

$$
f=f_{0} e_{0}+f_{1} e_{1}+f_{2} e_{2}+f_{3} e_{1} e_{2},
$$

for complex-valued functions $f_{j}, j=0,1,2,3$. Such a function $f$ is left monogenic if and only if it satisfies the following Cauchy-Riemann type system of differential equations:

$$
\begin{array}{ll}
\frac{\partial f_{0}}{\partial x_{0}}-\frac{\partial f_{1}}{\partial x_{1}}-\frac{\partial f_{2}}{\partial x_{2}}=0, & \frac{\partial f_{0}}{\partial x_{1}}+\frac{\partial f_{1}}{\partial x_{0}}+\frac{\partial f_{3}}{\partial x_{2}}=0 \\
\frac{\partial f_{0}}{\partial x_{2}}+\frac{\partial f_{2}}{\partial x_{0}}-\frac{\partial f_{3}}{\partial x_{1}}=0, & \frac{\partial f_{2}}{\partial x_{1}}-\frac{\partial f_{1}}{\partial x_{2}}+\frac{\partial f_{3}}{\partial x_{0}}=0
\end{array}
$$

For functions with values in the closed linear subspace $\mathbb{C}^{n+1}$ of $C \ell\left(\mathbb{C}^{n}\right)$, we have the following set of equivalent conditions.

Proposition 1.3 ([11, Proposition 1.7]). Let $f=f_{0} e_{0}-\sum_{j=1}^{n} f_{j} e_{j}$ be a $\mathbb{C}^{n+1}$-valued function defined on an open subset $U$ of $\mathbb{R}^{n+1}$. The following are equivalent:

(i) The $(n+1)$-tuple $F:=\left(f_{j}\right)_{j=0}^{n}$ is a system of complex conjugate harmonic functions in $U$, in the sense that it satisfies the generalised Cauchy-Riemann equations div $F=0$ and $\operatorname{curl} F=0$ in $U$.

(ii) $f$ is left monogenic in $U$.

(iii) $f$ is right monogenic in $U$.

(iv) The complex 1 -form $\omega:=f_{0} d x_{0}-f_{1} d x_{1}-\cdots-f_{n} d x_{n}$ satisfies $d \omega=0$ and $d^{*} \omega=0$ in $U$, where $d$ and $d^{*}$ are the exterior differentiation operator and its formal adjoint, respectively.

In addition, if the domain $U$ is simply connected, then the above conditions are further equivalent to the condition that

(v) there exists a unique (up to an additive constant) complex-valued harmonic function $V$ defined in $U$ such that $\left(f_{j}\right)_{j=0}^{n}=\operatorname{grad} V$ in $U$, that is, $f=\bar{D} V$.

1.3. The Cauchy integral formula. The Cauchy kernel is given by

$$
k(x-y)=\frac{1}{\sigma_{n}} \frac{\overline{x-y}}{|x-y|^{n+1}}, \quad x, y \in \mathbb{R}^{n+1}, x \neq y,
$$


with $\sigma_{n}=2 \pi^{(n+1) / 2} / \Gamma((n+1) / 2)$ the volume of the unit $n$-sphere in $\mathbb{R}^{n+1}$. The function $k$ is both left and right monogenic away from the origin. So, given a left monogenic function $f: U \rightarrow C \ell\left(\mathbb{C}^{n}\right)$ defined in an open subset $U$ of $\mathbb{R}^{n+1}$ and an open subset $\Omega$ of $U$ such that the closure $\bar{\Omega}$ of $\Omega$ is contained in $U$, and the boundary $\partial \Omega$ of $\Omega$ is a smooth oriented $n$-manifold, then the Cauchy integral formula

$$
f(y)=\int_{\partial \Omega} k(x-y) \boldsymbol{n}(x) f(x) d \mu(x), \quad y \in \Omega,
$$

is valid. Here $\boldsymbol{n}(x)$ is the outward unit normal at $x \in \partial \Omega$ and $\mu$ is the volume measure of the oriented manifold $\partial \Omega$. An element $x=\left(x_{0}, x_{1}, \ldots, x_{n}\right)$ of $\mathbb{R}^{n+1}$ will often be written as $x=x_{0} e_{0}+\boldsymbol{x}$ with $\boldsymbol{x}=\sum_{j=1}^{n} x_{j} e_{j}$. The expression $k(x-y)$ will also be written as $G_{x}(y)$-a more convenient notation when $y$ is replaced by an $n$-tuple of operators.

1.4. Monogenic extension. Because we shall be dealing with the monogenic extension into $\mathbb{R}^{n+1}$ of complex-valued real-analytic functions defined on open subsets of $\mathbb{R}^{n}$, it is worthwhile spelling out how this is done. More details are to be found from [2, Theorem 14.8]. Suppose that $f$ is an analytic $\mathbb{C}$-valued function defined on an open neighbourhood of zero in $\mathbb{R}^{n}$ and the Taylor series of $f$ is given by

$$
f(x)=\sum_{k=0}^{\infty} \frac{1}{k !} \sum_{l_{1}=1}^{n} \cdots \sum_{l_{k}=1}^{n} a_{l_{1} \ldots l_{k}} x_{l_{1}} \cdots x_{l_{k}}
$$

for all $x \in \mathbb{R}^{n}$ in a neighbourhood of zero. The coefficients $a_{l_{1} \ldots l_{k}} \in \mathbb{C}$ are assumed to be symmetric in $l_{1}, \ldots, l_{k}$. Expansions about other points $p$ in $\mathbb{R}^{n}$ are treated by translating $x \in \mathbb{R}^{n}$ to $x-p$.

Then the unique monogenic extension $\tilde{f}$ of $f$ is

$$
\widetilde{f}(x)=\sum_{k=0}^{\infty}\left(\sum_{\left(l_{1}, \ldots, l_{k}\right)} V^{l_{1} \ldots l_{k}}(x) a_{l_{1} \ldots l_{k}}\right)
$$

for all $x$ belonging to some neighbourhood of zero in $\mathbb{R}^{n+1}$. Here, the sum $\sum_{\left(l_{1}, \ldots, l_{k}\right)} \ldots$ is over the set $\left\{1 \leq l_{1} \leq \cdots \leq l_{k} \leq n\right\}$, and for $\left(l_{1}, \ldots, l_{k}\right) \in$ $\{1, \ldots, n\}^{k}$, the function $V^{l_{1} \ldots l_{k}}: \mathbb{R}^{n+1} \rightarrow C \ell\left(\mathbb{C}^{n}\right)$ is defined as follows. For each $j=1, \ldots, n$, the unique monogenic extension of the function $\boldsymbol{x}_{j}: x \mapsto$ $x_{j}, x \in \mathbb{R}^{n}$, is given by $z_{j}: x \mapsto x_{j} e_{0}-x_{0} e_{j}, x \in \mathbb{R}^{n+1}$. Then $V^{0}(x)=e_{0}$, $x \in \mathbb{R}^{n+1}$, and

$$
V^{l_{1} \ldots l_{k}}=\frac{1}{k !} \sum_{j_{1}, \ldots, j_{k}} \boldsymbol{z}_{j_{1}} \cdots \boldsymbol{z}_{j_{k}}
$$

where the sum is over all distinguishable permutations of all of $\left(l_{1}, \ldots, l_{k}\right)$, and products are in the sense of pointwise multiplication in $C \ell\left(\mathbb{C}^{n}\right)$. For- 
mula (6) also works if $f$ is $C \ell\left(\mathbb{C}^{n}\right)$-valued and $a_{l_{1} \ldots l_{k}} \in C \ell\left(\mathbb{C}^{n}\right)$. Then $\tilde{f}$ is left monogenic.

If $\widetilde{f}$ is left monogenic in the open ball $B_{R}(0)$ of radius $R$ about zero in $\mathbb{R}^{n+1}$, then (6) converges normally in $B_{R}(0)[2$, p. 82$]$.

The function $V^{l_{1} \ldots l_{k}}$ is both left and right monogenic. It is the unique monogenic $C \ell\left(\mathbb{C}^{n}\right)$-valued extension of the real-valued function $\boldsymbol{x}_{l_{1}} \cdots \boldsymbol{x}_{l_{k}}$ defined on $\mathbb{R}^{n}$ to all of $\mathbb{R}^{n+1}$, called the inner spherical monogenic polynomial [2, p. 68]. According to [2, Theorem 11.3.4, Remark 11.2.7(ii)], the monogenic function $V^{l_{1} \ldots l_{k}}$ actually takes its values in $\mathbb{R}^{n+1}$ although this is not immediately apparent from formula (7).

By locally extending power series like equation (5) to expansions like (6), any analytic function $f: U \rightarrow \mathbb{C}$ defined in an open subset of $\mathbb{R}^{n}$ is the restriction to $U$ of a unique function $\tilde{f}: V \rightarrow \mathbb{C}^{n+1}$ which is both left and right monogenic in an open subset $V$ of $\mathbb{R}^{n+1}$ such that $U=V \cap \mathbb{R}^{n}$ (see [2, Theorem 14.8, Remark 14.9]).

2. Joint spectral theory in the algebra $C \ell\left(\mathbb{C}^{n}\right)$. Let $\zeta=\left(\zeta_{1}, \ldots, \zeta_{n}\right)$ be a vector belonging to $\mathbb{C}^{n}$. The complex spectrum $\sigma(i \zeta)$ of the element $i \zeta=i\left(\zeta_{1} e_{1}+\cdots+\zeta_{n} e_{n}\right)$ of the algebra $C \ell\left(\mathbb{C}^{n}\right)$ is

$$
\sigma(i \zeta)=\left\{\lambda \in \mathbb{C}: \lambda e_{0}-i \zeta \text { does not have an inverse in } C \ell\left(\mathbb{C}^{n}\right)\right\} .
$$

Following [10, Section 5.2], we check that

$$
\left(\lambda e_{0}+i \zeta\right)\left(\lambda e_{0}-i \zeta\right)=\lambda^{2} e_{0}-i^{2} \zeta^{2}=\left(\lambda^{2}-|\zeta|_{\mathbb{C}}^{2}\right) e_{0}
$$

where $|\zeta|_{\mathbb{C}}^{2}=\sum_{j=1}^{n} \zeta_{j}^{2}$. So, for all $\lambda \in \mathbb{C}$ for which $\lambda \neq \pm|\zeta|_{\mathbb{C}}$, the element $\lambda e_{0}-i \zeta$ of the algebra $C \ell\left(\mathbb{C}^{n}\right)$ is invertible and

$$
\left(\lambda e_{0}-i \zeta\right)^{-1}=\frac{\lambda e_{0}+i \zeta}{\lambda^{2}-|\zeta|_{\mathbb{C}}^{2}}
$$

If $|\zeta|_{\mathbb{C}}^{2} \neq 0$, the two square roots of $|\zeta|_{\mathbb{C}}^{2}$ are written as $\pm|\zeta|_{\mathbb{C}}$, and $|\zeta|_{\mathbb{C}}=0$ for $|\zeta|_{\mathbb{C}}^{2}=0$. Hence $\sigma(i \zeta)=\left\{ \pm|\zeta|_{\mathbb{C}}\right\}$. When $|\zeta|_{\mathbb{C}}^{2} \neq 0$, the spectral projections

$$
\chi_{ \pm}(\zeta)=\frac{1}{2}\left(e_{0}+\frac{i \zeta}{ \pm|\zeta| \mathbb{C}}\right)
$$

are associated with each element $\pm|\zeta|_{\mathbb{C}}$ of the spectrum $\sigma(i \zeta)$ and $i \zeta$ has the spectral representation $i \zeta=|\zeta|_{\mathbb{C}} \chi_{+}(\zeta)+\left(-|\zeta|_{\mathbb{C}}\right) \chi_{-}(\zeta)$. In particular, for any function $\psi: \sigma(i \zeta) \rightarrow \mathbb{C}$, we set $\psi\{i \zeta\}=\psi\left(|\zeta|_{\mathbb{C}}\right) \chi_{+}(\zeta)+\psi\left(-|\zeta|_{\mathbb{C}}\right) \chi_{-}(\zeta)$.

Henceforth, we use the symbol $|\zeta|_{\mathbb{C}}$ to denote the square root of $|\zeta|_{\mathbb{C}}^{2}$ with positive real part in the case that $|\zeta|_{\mathbb{C}}^{2} \notin(-\infty, 0]$.

On the other hand, according to the point of view mentioned in the Introduction, for a complex vector $\zeta \in \mathbb{C}^{n}$, the monogenic spectrum $\gamma(\zeta)$ of the $n$-tuple $\zeta=\left(\zeta_{1}, \ldots, \zeta_{n}\right)$ of multiplication operators should be the set of singularities of the Cauchy kernel $x \mapsto G_{x}(\zeta), x \in \mathbb{R}^{n+1}$, in the algebra 
$C \ell\left(\mathbb{C}^{n}\right)$. Although $G_{x}(\zeta):=k(x-\zeta)$ is defined by formula (4) only for $\zeta \in \mathbb{R}^{n}$ and $x \neq \zeta$, a natural choice for the Cauchy kernel for $\zeta \in \mathbb{C}^{n}$ is to take the maximal holomorphic extension $\zeta \mapsto G_{x}(\zeta)$ of formula (4) for $\zeta \in \mathbb{C}^{n}$ :

$$
G_{x}(\zeta)=\frac{1}{\sigma_{n}} \frac{\bar{x}+\zeta}{|x-\zeta|_{\mathbb{C}}^{n+1}}, \quad x \in \mathbb{R}^{n+1}, \begin{cases}|x-\zeta|_{\mathbb{C}}^{2} \notin(-\infty, 0], & n \text { even } \\ |x-\zeta|_{\mathbb{C}}^{2} \neq 0, & n \text { odd }\end{cases}
$$

Here $|x-\zeta|_{\mathbb{C}}^{2}=x_{0}^{2}+\sum_{j=1}^{n}\left(x_{j}-\zeta_{j}\right)^{2}$ for $x=\left(x_{0}, x_{1}, \ldots, x_{n}\right) \in \mathbb{R}^{n+1}$ and the complex number $|x-\zeta|_{\mathbb{C}}$ is the positive square root of $|x-\zeta|_{\mathbb{C}}^{2}$, coinciding with the holomorphic extension of the modulus function $\xi \mapsto|x-\xi|, \xi \in \mathbb{R}^{n} \backslash\{x\}$, in the case $x \in \mathbb{R}^{n}$. There is a discontinuity in the function $(x, \zeta) \mapsto|x-\zeta|_{\mathbb{C}}$ on the set

$$
\left\{(x, \zeta) \in \mathbb{R}^{n+1} \times \mathbb{C}^{n}:|x-\zeta|_{\mathbb{C}}^{2} \in(-\infty, 0]\right\} .
$$

The analogous reasoning for multiplication by $x \in \mathbb{R}^{n+1}$ in the algebra $C \ell\left(\mathbb{C}^{n}\right)$ just gives us the Cauchy kernel (4), so that $\gamma(x)=\{x\}$, as expected.

Given $\zeta \in \mathbb{C}^{n}$, if singularities of (9) occur at $x \in \mathbb{R}^{n+1}$, then $|x-\zeta|_{\mathbb{C}}^{2} \in$ $(-\infty, 0]$, otherwise we can simply take the positive square root of $|x-\zeta|_{\mathbb{C}}^{2}$ in formula (9) to obtain a monogenic function of $x$. To determine the set of $x \in \mathbb{R}^{n+1}$ where singularities occur, write $\zeta=\xi+i \eta$ for $\xi, \eta \in \mathbb{R}^{n}$ and $x=x_{0} e_{0}+\boldsymbol{x}$ for $x_{0} \in \mathbb{R}$ and $\boldsymbol{x} \in \mathbb{R}^{n}$. Then

$$
\begin{aligned}
|x-\zeta|_{\mathbb{C}}^{2} & =x_{0}^{2}+\sum_{j=1}^{n}\left(x_{j}-\zeta_{j}\right)^{2}=x_{0}^{2}+\sum_{j=1}^{n}\left(x_{j}-\xi_{j}-i \eta_{j}\right)^{2} \\
& =x_{0}^{2}+|\boldsymbol{x}-\xi|^{2}-|\eta|^{2}-2 i\langle\boldsymbol{x}-\xi, \eta\rangle .
\end{aligned}
$$

Thus, $|x-\zeta|_{\mathbb{C}}^{2}$ belongs to $(-\infty, 0]$ if and only if $x$ lies in the intersection of the hyperplane $\langle\boldsymbol{x}-\xi, \eta\rangle=0$ passing through $\xi$ and with normal $\eta$, and the ball $x_{0}^{2}+|\boldsymbol{x}-\xi|^{2} \leq|\eta|^{2}$ centred at $\xi$ with radius $|\eta|$. If $n$ is even, then

$$
\gamma(\zeta)=\left\{x=x_{0} e_{0}+\boldsymbol{x} \in \mathbb{R}^{n+1}:\langle\boldsymbol{x}-\xi, \eta\rangle=0, x_{0}^{2}+|\boldsymbol{x}-\xi|^{2} \leq|\eta|^{2}\right\} .
$$

and if $n$ is odd, then

$$
\gamma(\zeta)=\left\{x=x_{0} e_{0}+\boldsymbol{x} \in \mathbb{R}^{n+1}:\langle\boldsymbol{x}-\xi, \eta\rangle=0, x_{0}^{2}+|\boldsymbol{x}-\xi|^{2}=|\eta|^{2}\right\} .
$$

In particular, if $\Im(\zeta)=0$, then $\gamma(\zeta)=\{\zeta\} \subset \mathbb{R}^{n}$.

Off $\gamma(\zeta)$, a calculation shows that the function $x \mapsto G_{x}(\zeta)$ is two-sided monogenic, so the Cauchy integral formula gives

$$
\widetilde{f}(\zeta)=\int_{\partial \Omega} G_{x}(\zeta) \boldsymbol{n}(x) f(x) d \mu(x)
$$

for a bounded open neighbourhood $\Omega$ of $\gamma(\zeta)$ with smooth oriented boundary $\partial \Omega$, outward unit normal $\boldsymbol{n}(x)$ at $x \in \partial \Omega$ and surface measure $\mu$. The function $f$ is assumed to be left monogenic in a neighbourhood of $\bar{\Omega}$, but $\zeta \mapsto \widetilde{f}(\zeta)$ is a holomorphic $C \ell\left(\mathbb{C}^{n}\right)$-valued function as the closed set $\gamma(\zeta)$ 
varies inside $\Omega$. Moreover, $\tilde{f}$ equals $f$ on $\Omega \cap \mathbb{R}^{n}$ by the usual Cauchy integral formula of Clifford analysis, so if $f$ is, say, the monogenic extension of a polynomial $p: \mathbb{C}^{n} \rightarrow \mathbb{C}$ restricted to $\mathbb{R}^{n}$, then $\tilde{f}(\zeta)=p(\zeta)$, as expected. In this way, with each left monogenic function $f$ defined in a neighbourhood of $\gamma(\zeta)$, in a natural way we associate a holomorphic function $\widetilde{f}$ defined in a neighbourhood of $\zeta$.

For each $0<\omega<\pi / 2$, we set

$$
\begin{aligned}
& S_{\omega}\left(\mathbb{R}^{n+1}\right)=\left\{x \in \mathbb{R}^{n+1}: x=x_{0} e_{0}+\boldsymbol{x},\left|x_{0}\right| \leq|\boldsymbol{x}| \tan \omega\right\}, \\
& S_{\omega}^{\circ}\left(\mathbb{R}^{n+1}\right)=\left\{x \in \mathbb{R}^{n+1} \backslash\{0\}: x=x_{0} e_{0}+\boldsymbol{x},\left|x_{0}\right|<|\boldsymbol{x}| \tan \omega\right\} .
\end{aligned}
$$

It is clear that if $\zeta=\xi+i \eta$ lies in a sector in $\mathbb{C}^{n}$, say, $|\eta| \leq|\xi| \tan \nu$, then the monogenic spectrum $\gamma(\zeta)$ lies in a corresponding sector in $\mathbb{R}^{n+1}$. More precisely, we have

Proposition 2.1. Let $\zeta \in \mathbb{C}^{n} \backslash\{0\}$ and $0<\omega<\pi / 2$. Then $\gamma(\zeta) \subset$ $S_{\omega}\left(\mathbb{R}^{n+1}\right)$ if and only if

$$
|\zeta|_{\mathbb{C}}^{2} \neq(-\infty, 0], \quad|\Im(\zeta)| \leq \Re\left(|\zeta|_{\mathbb{C}}\right) \tan \omega .
$$

Proof. The statement is trivially valid if $\zeta \in \mathbb{R}^{n} \backslash\{0\}$, so suppose that $\Im(\zeta) \neq 0$. Then the monogenic spectrum $\gamma(\zeta)$ of $\zeta$ given by (11) or (12) is a subset of $S_{\omega}\left(\mathbb{R}^{n+1}\right)$ if and only if there exists $0<\theta \leq \omega$ such that the cone

$$
H_{\theta}^{+}=\left\{x=x_{0} e_{0}+\boldsymbol{x} \in \mathbb{R}^{n+1}: x_{0}>0, x_{0}=|x| \tan \theta\right\}
$$

is tangential to the boundary of $\gamma(\zeta)$. A calculation shows that $H_{\theta}^{+}$is tangential to the boundary of $\gamma(\zeta)$ for all $\zeta=\xi+i \eta$ with $\xi, \eta \in \mathbb{R}^{n}$, satisfying

$$
|\eta|^{2}=\sin ^{2} \theta \cdot\left(|\xi|^{2}+\left|P_{\eta} \xi\right|^{2} \tan ^{2} \theta\right) .
$$

Here $P_{\eta}: u \mapsto\langle u, \eta\rangle \eta /|\eta|^{2}, u \in \mathbb{R}^{n}$, is the projection operator onto $\operatorname{span}\{\eta\}$.

To relate condition (16) to the inequality in (14), suppose that $m=$ $m_{0} e_{0}+\boldsymbol{m}$ is the unit vector normal to $H_{\theta}$ such that $\boldsymbol{m}$ lies in the direction of $\eta$. Hence, $m_{0}=\cot \theta|\boldsymbol{m}|, \tan \theta=|\boldsymbol{m}| / m_{0}$ and $P_{\eta} \xi=\langle\xi, \boldsymbol{m}\rangle \boldsymbol{m} /|\boldsymbol{m}|^{2}$. Then equation (16) becomes

$$
\eta=\sin \theta \cdot\left(m_{0}^{2}|\xi|^{2}+\langle\xi, \boldsymbol{m}\rangle^{2}\right)^{1 / 2} \frac{\boldsymbol{m}}{|\boldsymbol{m}| m_{0}} .
$$

But $\left|m_{0} e_{0}+\boldsymbol{m}\right|=1$, so $\left(\cot ^{2} \theta+1\right)|\boldsymbol{m}|^{2}=1$. We have $|\boldsymbol{m}|=\sin \theta$ and

$$
\eta=\left(m_{0}^{2}|\xi|^{2}+\langle\xi, \boldsymbol{m}\rangle^{2}\right)^{1 / 2} \frac{\boldsymbol{m}}{m_{0}} .
$$

As mentioned in [10, p. 67], the set of all $\zeta=\xi+i \eta$ with $\eta \neq 0$ satisfying (17) is equal to the set of all $\zeta=\xi+i \eta$ with $\eta \neq 0$ satisfying

$$
|\zeta|_{\mathbb{C}}^{2} \neq(-\infty, 0], \quad \eta=\Re\left(|\zeta|_{\mathbb{C}}\right) \frac{\boldsymbol{m}}{m_{0}} .
$$

Because $|\boldsymbol{m}| / m_{0}=\tan \theta \leq \tan \omega$, we obtain the desired equivalence by letting $\boldsymbol{m}$ vary over all directions in $\mathbb{R}^{n}$. 
For each $0<\omega<\pi / 2$, let $S_{\omega}\left(\mathbb{C}^{n}\right)$ denote the set of all $\zeta \in \mathbb{C}^{n}$ satisfying condition (14) and let $S_{\omega}^{\circ}\left(\mathbb{C}^{n}\right)$ be its interior. The following results are elementary consequences of the integral representation (13) and Proposition 2.1.

Corollary 2.2. Let $0<\omega<\pi / 2$, let $f: S_{\omega}^{\circ}\left(\mathbb{R}^{n+1}\right) \rightarrow C \ell\left(\mathbb{C}^{n}\right)$ be a left monogenic function and suppose that $\tilde{f}: S_{\omega}^{\circ}\left(\mathbb{C}^{n}\right) \rightarrow C \ell\left(\mathbb{C}^{n}\right)$ is defined by formula (13) for every $\zeta \in S_{\omega}^{\circ}\left(\mathbb{C}^{n}\right)$ with $\Omega$ chosen such that $\gamma(\zeta) \subset \Omega \subset$ $\bar{\Omega} \subset S_{\omega}^{\circ}\left(\mathbb{R}^{n+1}\right)$. Then $\zeta \mapsto \widetilde{f}(\zeta), \zeta \in S_{\omega}^{\circ}\left(\mathbb{C}^{n}\right)$, is a holomorphic $C \ell\left(\mathbb{C}^{n}\right)$-valued function equal to $f$ on $\mathbb{R}^{n} \backslash\{0\}$. If $K$ is a compact subset of $S_{\omega}^{\circ}\left(\mathbb{C}^{n}\right)$ and $\Omega$ is a bounded open neighbourhood of $\bigcup_{\zeta \in K} \gamma(\zeta)$ with smooth oriented boundary $\partial \Omega$ such that $\bar{\Omega} \subset S_{\omega}^{\circ}\left(\mathbb{R}^{n+1}\right)$, then there exists $C_{K, \Omega}>0$, independent of $f$, such that

$$
\sup _{\zeta \in K}|\widetilde{f}(\zeta)| \leq C_{K, \Omega} \sup _{\omega \in \partial \Omega}|f(\omega)| .
$$

Corollary 2.3. Let $0<\omega<\pi / 2$ and let $f: S_{\omega}^{\circ}\left(\mathbb{R}^{n+1}\right) \rightarrow C \ell\left(\mathbb{C}^{n}\right)$ be a left monogenic function. Denote the restriction of $f$ to $\mathbb{R}^{n} \backslash\{0\}$ by $f_{\mathbb{R}^{n}}$. Then $f_{\mathbb{R}^{n}}$ is the restriction to $\mathbb{R}^{n} \backslash\{0\}$ of a holomorphic $C \ell\left(\mathbb{C}^{n}\right)$-valued function $\widetilde{f}$ defined on $S_{\omega}^{\circ}\left(\mathbb{C}^{n}\right)$. If $f_{\mathbb{R}^{n}}$ takes values in $\mathbb{C}$, then so does $\widetilde{f}$.

Proof. According to Proposition 2.1, formula (13) defines a holomorphic function $\tilde{f}: S_{\omega}^{\circ}\left(\mathbb{C}^{n}\right) \rightarrow C \ell\left(\mathbb{C}^{n}\right)$, because for every $\zeta \in S_{\omega}^{\circ}\left(\mathbb{C}^{n}\right)$, the function $f$ is left monogenic in a neighbourhood of the monogenic spectrum $\gamma(\zeta)$ of $\zeta$. Moreover, $\widetilde{f}(x)=f_{0}(x)$ for all nonzero $x \in \mathbb{R}^{n}$, because $\gamma(x)=\{x\}$ and the Cauchy integral formula of Clifford analysis holds. By unique continuation, $\widetilde{f}$ takes values in the same subspace of $C \ell\left(\mathbb{C}^{n}\right)$ in which $f_{0}$ does.

The sector $S_{\omega}\left(\mathbb{C}^{n}\right)$ arose in [8] as the set of $\zeta \in \mathbb{C}^{n}$ for which the exponential functions

$$
e_{+}(x, \zeta)=e^{i\langle\boldsymbol{x}, \zeta\rangle} e^{-x_{0}|\zeta| \mathbb{c}} \chi_{+}(\zeta), \quad x=x_{0} e_{0}+\boldsymbol{x},
$$

have decay at infinity for all $x \in \mathbb{R}^{n+1}$ with $\langle x, m\rangle>0$ and all unit vectors $m=m_{0} e_{0}+\boldsymbol{m} \in \mathbb{R}^{n+1}$ satisfying $m_{0} \geq|\boldsymbol{m}| \cot \omega$; this fact is essential for the arguments of Section 4. The projection $\chi_{+}$in formula (19) is defined by equation (8).

Let $0<\nu<\pi / 2$ and let $H_{\ell}^{\infty}\left(S_{\nu}^{\circ}\left(\mathbb{R}^{n+1}\right)\right)$ denote the set of all left monogenic functions $f: S_{\nu}^{\circ}\left(\mathbb{R}^{n+1}\right) \rightarrow C \ell\left(\mathbb{C}^{n}\right)$ that are uniformly bounded on every subsector $S_{\nu^{\prime}}^{\circ}\left(\mathbb{R}^{n+1}\right), 0<\nu^{\prime}<\nu$. Endowed with the topology of uniform convergence on subsectors $S_{\nu^{\prime}}^{\circ}\left(\mathbb{R}^{n+1}\right), 0<\nu^{\prime}<\nu$, the topological vector space $H_{\ell}^{\infty}\left(S_{\nu}^{\circ}\left(\mathbb{R}^{n+1}\right)\right)$ is a Fréchet space. The analogous space for right monogenic functions is written as $H_{r}^{\infty}\left(S_{\nu}^{\circ}\left(\mathbb{R}^{n+1}\right)\right)$.

For each $0<\nu<\pi / 2$, let $H^{\infty}\left(S_{\nu}^{\circ}\left(\mathbb{C}^{n}\right)\right)$ denote the set of all holomorphic functions $f: S_{\nu}^{\circ}\left(\mathbb{C}^{n}\right) \rightarrow C \ell\left(\mathbb{C}^{n}\right)$ which are uniformly bounded on every 
subsector $S_{\nu^{\prime}}^{\circ}\left(\mathbb{C}^{n}\right), 0<\nu^{\prime}<\nu$. Endowed with the topology of uniform convergence on subsectors $S_{\nu^{\prime}}^{\circ}\left(\mathbb{C}^{n}\right), 0<\nu^{\prime}<\nu$, the topological vector space $H^{\infty}\left(S_{\nu}^{\circ}\left(\mathbb{C}^{n}\right)\right)$ is a Fréchet space and a (nonabelian) Fréchet algebra under pointwise multiplication. The subalgebra of $\mathbb{C}$-valued functions is, of course, an abelian Fréchet algebra.

The main aim of the present paper is to prove the following function space isomorphism.

THEOREM 2.4. The mapping $f \mapsto \widetilde{f}$ given by the Cauchy integral formula (13) is an isomorphism of the Fréchet spaces $H_{\ell}^{\infty}\left(S_{\nu}^{\circ}\left(\mathbb{R}^{n+1}\right)\right)$ and $H^{\infty}\left(S_{\nu}^{\circ}\left(\mathbb{C}^{n}\right)\right)$.

Given two functions $f, g \in H^{\infty}\left(S_{\nu}^{\circ}\left(\mathbb{R}^{n+1}\right)\right)$, the restrictions $f_{\mathbb{R}^{n}}: \mathbb{R}^{n} \backslash\{0\}$ $\rightarrow C \ell\left(\mathbb{C}^{n}\right)$ and $g_{\mathbb{R}^{n}}: \mathbb{R}^{n} \backslash\{0\} \rightarrow C \ell\left(\mathbb{C}^{n}\right)$ of $f$ and $g$ to $\mathbb{R}^{n} \backslash\{0\}$ are realanalytic, so their product $f_{\mathbb{R}^{n}} g_{\mathbb{R}^{n}}$ has a unique left monogenic extension $f \cdot \ell g$ to an open neighbourhood of $\mathbb{R}^{n} \backslash\{0\}$ in $\mathbb{R}^{n+1}$ by virtue of formula (6). The analogous product for right monogenic functions is written as $f \cdot_{r} g$.

Corollary 2.5. For each $f, g \in H^{\infty}\left(S_{\nu}^{\circ}\left(\mathbb{R}^{n+1}\right)\right)$, the function $f \cdot \ell g$ has a left monogenic extension to $S_{\nu}^{\circ}\left(\mathbb{R}^{n+1}\right)$, denoted by the same symbol, and $f \cdot \ell g \in H^{\infty}\left(S_{\nu}^{\circ}\left(\mathbb{R}^{n+1}\right)\right)$. Moreover, $H_{\ell}^{\infty}\left(S_{\nu}^{\circ}\left(\mathbb{R}^{n+1}\right)\right)$ is a Fréchet algebra with the product $(f, g) \mapsto f \cdot \ell g$. The mapping $f \mapsto \widetilde{f}$ given by the Cauchy integral formula (13) is an isomorphism of the Fréchet algebras $H_{\ell}^{\infty}\left(S_{\nu}^{\circ}\left(\mathbb{R}^{n+1}\right)\right)$ and $H^{\infty}\left(S_{\nu}^{\circ}\left(\mathbb{C}^{n}\right)\right)$.

Proof. By Theorem 2.4, the unique left monogenic extension $f \cdot \ell g$ to an open neighbourhood of $\mathbb{R}^{n} \backslash\{0\}$ in $\mathbb{R}^{n+1}$ has the property that $f_{\mathbb{R}^{n}} g_{\mathbb{R}^{n}}$ is the restriction to $\mathbb{R}^{n} \backslash\{0\}$ of the $C \ell\left(\mathbb{C}^{n}\right)$-valued holomorphic function $\widetilde{f} \cdot \widetilde{g}$ belonging to $H^{\infty}\left(S_{\nu}^{\circ}\left(\mathbb{C}^{n}\right)\right)$. But by Theorem $2.4, \tilde{f} \cdot \widetilde{g}$ is the image of some function belonging to $H^{\infty}\left(S_{\nu}^{\circ}\left(\mathbb{R}^{n+1}\right)\right)$. By unique continuation of monogenic functions (see Subsection 1.4), this function must be equal to $f \cdot \ell g$ on their common domain, that is, $f \cdot \ell g$ has a unique left monogenic extension to $S_{\nu}^{\circ}\left(\mathbb{R}^{n+1}\right)$ belonging to $H^{\infty}\left(S_{\nu}^{\circ}\left(\mathbb{R}^{n+1}\right)\right)$.

If the restrictions $f_{\mathbb{R}^{n}}, g_{\mathbb{R}^{n}}$ of $f$ and $g$ take values in $\mathbb{C}$ rather than $C \ell\left(\mathbb{C}^{n}\right)$, then both $f$ and $g$ are two-sided monogenic and $\left(f \cdot_{r} g\right)^{\sim}=(f \cdot \ell g)^{\sim}=\widetilde{f} \cdot \widetilde{g}$ $=\widetilde{g} \cdot \tilde{f}$. Hence, $f \cdot \ell g=g \cdot \ell f$ and the subalgebra of $H_{\ell}^{\infty}\left(S_{\nu}^{\circ}\left(\mathbb{R}^{n+1}\right)\right)$ consisting of such functions is abelian.

3. Bounded monogenic functions in sectors. In this section we suppose that $0<\nu<\pi / 2$ and $f: S_{\nu}^{\circ}\left(\mathbb{R}^{n+1}\right) \rightarrow C \ell\left(\mathbb{C}^{n}\right)$ is a left monogenic function that is uniformly bounded in $S_{\nu}^{\circ}\left(\mathbb{R}^{n+1}\right)$. Denote the supremum of $|f(x)|$ for $x \in S_{\nu}^{\circ}\left(\mathbb{R}^{n+1}\right)$ by $\|f\|_{\nu, \infty}$.

According to Corollary 2.3, for every $0<\nu^{\prime}<\nu$, there exists a holomorphic function $\widetilde{f}: S_{\nu^{\prime}}^{\circ}\left(\mathbb{C}^{n}\right) \rightarrow C \ell\left(\mathbb{C}^{n}\right)$ coinciding with $f$ on $\mathbb{R}^{n}$. By analytic 
continuation, $\widetilde{f}$ takes its values in the subspace spanned by the range of $f$ on $\mathbb{R}^{n}$. We are aiming to bound the uniform norm of $\widetilde{f}$ on $S_{\nu^{\prime}}^{\circ}\left(\mathbb{C}^{n}\right)$ in terms of a uniform bound for $f$ on $S_{\nu}^{\circ}\left(\mathbb{R}^{n+1}\right)$.

We can easily find such a bound on a smaller sector in $S_{\nu}\left(\mathbb{C}^{n}\right)$. Let $0<\nu^{\prime}<\pi / 2$ and let $\widetilde{S}_{\nu^{\prime}}\left(\mathbb{C}^{n}\right)$ denote the set of all $\zeta \in \mathbb{C}^{n}$ such that $|\eta| \leq|\xi| \sin \nu^{\prime}$ for $\zeta=\xi+i \eta, \xi, \eta \in \mathbb{R}^{n}$. Then for each $\zeta \in \widetilde{S}_{\nu^{\prime}}\left(\mathbb{C}^{n}\right)$ with $\zeta=\xi+i \eta$ for $\xi, \eta \in \mathbb{R}^{n}$, the closed ball of radius $|\eta|$ centred at $\xi$ in $\mathbb{R}^{n+1}$ is contained in $S_{\nu^{\prime}}\left(\mathbb{R}^{n+1}\right)$. For this to be true, necessarily $|\eta|<|\xi|$, which does not hold for all $\zeta \in S_{\nu^{\prime}}\left(\mathbb{C}^{n}\right)$.

Now let $0<\nu^{\prime}<\theta<\nu$. If $\zeta \in \widetilde{S}_{\nu^{\prime}}\left(\mathbb{C}^{n}\right)$, then the closed ball $B_{\zeta, \delta}$ of radius $|\eta|(1+\delta)$ centred at $\xi$ strictly contains $\gamma(\zeta)$ and is contained in $S_{\theta}\left(\mathbb{R}^{n+1}\right) \backslash\{0\} \subset S_{\nu}^{\circ}\left(\mathbb{R}^{n+1}\right)$, provided that $0<\delta \leq \sin \theta / \sin \nu^{\prime}-1$ and then it follows that

$$
\widetilde{f}(\zeta)=\int_{\partial B_{\zeta, \delta}} G_{x}(\zeta) \boldsymbol{n}(x) f(x) d \mu(x) .
$$

Then $|\widetilde{f}(\zeta)| \leq 2^{n / 2}\|f\|_{\nu, \infty} \int_{\partial B_{\zeta, \delta}}\left|G_{x}(\zeta)\right| d \mu(x)$, so we need to estimate $\left|G_{x}(\zeta)\right|$ for $x \in B_{\zeta, \delta}$ and $\zeta \in \widetilde{S}_{\nu^{\prime}}\left(\mathbb{C}^{n}\right)$. To this end,

$$
\frac{\bar{x}+\zeta}{|x-\zeta|_{\mathbb{C}}^{n+1}}=\frac{1}{|\zeta|^{n}} \frac{\bar{x} /|\zeta|+\zeta /|\zeta|}{\left.|x /| \zeta|-\zeta /| \zeta\right|_{\mathbb{C}} ^{n+1}}
$$

Every $x \in B_{\zeta, \delta}$ can be written as $x=\xi+|\eta|(1+\delta) \alpha$ for $\alpha \in S^{n}$, the unit sphere in $\mathbb{R}^{n+1}$, so that $x /|\zeta|=\xi /|\zeta|+\alpha|\eta|(1+\delta) /|\zeta|$. It turns out that the numbers $|\zeta|$ and $|\xi|$ are comparable for $\zeta \in S_{\nu^{\prime}}\left(\mathbb{C}^{n}\right)$ and $|\eta|$ is dominated by a $|\zeta|$. Because $\mu\left(B_{\zeta, \delta}\right)=O\left(|\zeta|^{n}\right)$ as $\zeta$ goes to infinity or zero, we obtain a uniform bound on $\int_{\partial B_{\zeta, \delta}}\left|G_{x}(\zeta)\right| d \mu(x)$ for $\zeta \in \widetilde{S}_{\nu^{\prime}}\left(\mathbb{C}^{n}\right)$. The bound depends only on $\nu^{\prime}, \delta$ and $n$.

If we want to extend this bound from the sector $\widetilde{S}_{\nu^{\prime}}\left(\mathbb{C}^{n}\right)$ to all of $S_{\nu^{\prime}}\left(\mathbb{C}^{n}\right)$, then we need to take into account the geometry of the situation, that is, the difficulty with fitting a ball into a sector in $\mathbb{R}^{n+1}$. The obvious remedy is to replace the ball $B_{\zeta, \delta}$ by a suitable disk about $\gamma(\zeta)$. We now work out the details of the approach outlined above for this case.

Given $\zeta \in S_{\nu^{\prime}}\left(\mathbb{C}^{n}\right)$, write $\zeta=\xi+i \eta$ for $\xi, \eta \in \mathbb{R}^{n}$. Suppose that $\eta \neq 0$. For each $\delta>0$, let $D_{\zeta, \delta}$ denote the right hypercylinder in $\mathbb{R}^{n+1} \equiv \mathbb{R} \times \mathbb{R}^{n}$ centred at $(0, \xi)$ with radius $|\eta|(1+\delta)$ and bounded by the hyperplanes

$$
P_{ \pm}=\left\{x \in \mathbb{R}^{n+1}: x=x_{0} e_{0}+\boldsymbol{x},\langle\boldsymbol{x}-(\xi \pm \delta \eta / 2), \eta\rangle=0\right\} .
$$

Then the monogenic spectrum $\gamma(\zeta)$ of $\zeta$ is contained in $D_{\zeta, \delta}$ and $\mu\left(\partial D_{\zeta, \delta}\right)=$ $O\left(|\eta|^{n}\right)$ as $\zeta$ goes to zero or infinity in $S_{\nu^{\prime}}\left(\mathbb{C}^{n}\right)$, for if $\Sigma_{n}$ is the $n$-volume of the unit ball $\left\{x \in \mathbb{R}^{n}:|x| \leq 1\right\}$ in $\mathbb{R}^{n}$ and $\sigma_{n-1}$ is the $(n-1)$-volume of the 
hypersphere $\left\{x \in \mathbb{R}^{n}:|x|=1\right\}$ in $\mathbb{R}^{n}$, then

$$
\mu\left(\partial D_{\zeta, \delta}\right)=2 \Sigma_{n}|\eta|^{n}(1+\delta)^{n}+\sigma_{n-1}|\eta|^{n-1}(1+\delta)^{n-1} \delta|\eta| .
$$

Of course, $D_{\zeta, \delta} \rightarrow\{\xi\}$ as $\eta \rightarrow 0$.

LEMma 3.1. For each $\delta>0$, there exists $\varepsilon_{\delta}>0$ depending only on $\delta$ and $n$ such that ||$x-\left.\zeta\right|_{\mathbb{C}}\left|\geq \varepsilon_{\delta}\right| \zeta \mid$ for all $x \in \partial D_{\zeta, \delta}$ and $\zeta \in \mathbb{C}^{n}$ with $\Im \zeta \neq 0$.

Proof. Because $D_{t \zeta, \delta}=t D_{\zeta, \delta}$ for all $t>0$ and the function $|\cdot|_{\mathbb{C}}$ is homogeneous of degree one, it is enough to prove the statement for all $\zeta \in \mathbb{C}^{n}$ with $|\zeta|=1$.

Let $S^{n-1}\left(\mathbb{C}^{n}\right)$ be the set of all $\zeta \in \mathbb{C}^{n}$ with $|\zeta|=1$. Given $\zeta \in S^{n-1}\left(\mathbb{C}^{n}\right)$ with $\zeta=\xi+i \eta$ for $\xi \in \mathbb{R}^{n}$ and $\eta \in \mathbb{R}^{n} \backslash\{0\}$, every element of $\mathbb{R}^{n+1}$ belonging to the intersection of $\partial D_{\zeta, \delta}$ with the hyperplane $P_{+}$can be parametrised as $x(\zeta, r, \alpha)=\xi+\delta \eta / 2+|\eta|(1+\delta) r T_{\eta} \alpha$ for $0 \leq r \leq 1$ and $\alpha$ belonging to the unit sphere $S^{n-1}$ in $\mathbb{R}^{n}$. Here $T_{\eta}$ is a rotation mapping $\mathbb{R}^{n}$ onto the $n$-dimensional subspace $\{\eta\}^{\perp}$ of $\mathbb{R}^{n+1}$ such that $\eta \mapsto T_{\eta}$ is continuous on $S^{n-1}$. Then $x(\zeta, r, \alpha) \notin \gamma(\zeta)$, so $|x(\zeta, r, \alpha)-\zeta|_{\mathbb{C}}$ is nonzero. The function $(\zeta, r, \alpha) \mapsto|| x(\zeta, r, \alpha)-\left.\zeta\right|_{\mathbb{C}} \mid$ is positive and continuous on the compact set $S^{n-1}\left(\mathbb{C}^{n}\right) \times[0,1] \times S^{n-1}$ and so

$$
\inf \left\{|| x(\zeta, r, \alpha)-\left.\zeta\right|_{\mathbb{C}} \mid:(\zeta, r, \alpha) \in S^{n-1}\left(\mathbb{C}^{n}\right) \times[0,1] \times S^{n-1}\right\}
$$

is a strictly positive number. A similar argument works for the other face intersecting $P_{-}$.

On the third face, the parametrisation $x(\zeta, t, \alpha)=\xi+\delta \eta t / 2+|\eta|(1+\delta) T_{\eta} \alpha$ for $-1 \leq t \leq 1$ and $\alpha$ belonging to the unit sphere $S^{n-1}$ is used. The required number $\varepsilon_{\delta}$ is the minimum of these three numbers.

REMARK 3.2. The expression ||$x(\zeta, r, \alpha)-\left.\left.\zeta\right|_{\mathbb{C}} ^{2}\right|^{2}$ is quartic in the $3 n+1$ real variables $\xi, \eta, \beta$ and $r$, if we take $\beta$ to represent $T_{\eta} \alpha$. Estimating $\varepsilon_{\delta}$ is likely to be unrewarding.

Lemma 3.3. For each $\delta>0$, let $\varepsilon_{\delta}>0$ be the number given in Lemma 3.1. Then

$$
\int_{\partial D_{\zeta, \delta}}\left|G_{x}(\zeta)\right| d \mu(x) \leq \frac{3(1+\delta / 2)(1+\delta)^{n}\left(2 \Sigma_{n}+\sigma_{n-1} \delta\right)}{\sigma_{n} \varepsilon_{\delta}^{n+1}}
$$

for all $\zeta \in \mathbb{C}^{n}$ with $\Im \zeta \neq 0$.

Proof. If $x \in \partial D_{\zeta, \delta}$ and $\zeta \in \mathbb{C}^{n}$ with $\Im \zeta \neq 0$, then

$$
\begin{aligned}
\left|G_{x}(\zeta)\right| & \leq \frac{1}{\sigma_{n}|\zeta|^{n}} \frac{|\bar{x} /| \zeta|+\zeta /| \zeta||}{|| x /|\zeta|-\zeta /|\zeta|_{\mathbb{C}}^{n+1} \mid} \leq \frac{1}{\sigma_{n}|\zeta|^{n}} \frac{|\bar{x}| /|\zeta|+1}{|| x /|\zeta|-\zeta /\left.|\zeta|_{\mathbb{C}}\right|^{n+1}} \\
& \leq \frac{1}{\sigma_{n}|\zeta|^{n}} \frac{|\bar{x}| / \zeta \mid+1}{\varepsilon_{\delta}^{n+1}}
\end{aligned}
$$


by Lemma 3.1. For $x \in \partial D_{\zeta, \delta}$, we have $|\bar{x}| /|\zeta| \leq 2+3 \delta / 2$ and from equation (20), we obtain

$$
\frac{\mu\left(\partial D_{\zeta, \delta}\right)}{|\zeta|^{n}} \leq 2 \Sigma_{n}(1+\delta)^{n}+\sigma_{n-1}(1+\delta)^{n-1} \delta
$$

Combining these estimates gives the stated inequality.

The next result improves Corollary 2.2 in the context of sectors and proves the first half of Theorem 2.4.

Lemma 3.4. Let $0<\nu<\pi / 2$ and let $f: S_{\nu}^{\circ}\left(\mathbb{R}^{n+1}\right) \rightarrow C \ell\left(\mathbb{C}^{n}\right)$ be a uniformly bounded left monogenic function. Suppose that $\widetilde{f}: S_{\nu}^{\circ}\left(\mathbb{C}^{n}\right) \rightarrow$ $C \ell\left(\mathbb{C}^{n}\right)$ is defined by formula (13) for every $\zeta \in S_{\nu}^{\circ}\left(\mathbb{C}^{n}\right)$ with $\Omega$ chosen such that $\gamma(\zeta) \subset \Omega \subset \bar{\Omega} \subset S_{\nu}^{\circ}\left(\mathbb{R}^{n+1}\right)$. Then for every $0<\nu^{\prime}<\nu$, the function $\zeta \mapsto \widetilde{f}(\zeta), \zeta \in S_{\nu^{\prime}}^{\circ}\left(\mathbb{C}^{n}\right)$, is a uniformly bounded holomorphic $C \ell\left(\mathbb{C}^{n}\right)$-valued function equal to $f$ on $\mathbb{R}^{n} \backslash\{0\}$. It is bounded by $\|f\|_{\nu, \infty}$ times a constant $C$ depending only on $n, \nu^{\prime}$ and $\nu$.

Proof. Let $0<\nu^{\prime}<\theta<\nu$. According to Proposition 2.1, $\gamma(\zeta) \subset$ $S_{\nu^{\prime}}^{\circ}\left(\mathbb{R}^{n+1}\right)$ for all $\zeta \in S_{\nu^{\prime}}\left(\mathbb{C}^{n}\right)$. We can choose $\delta>0$ such that $D_{\zeta, \delta} \subset$ $S_{\theta}\left(\mathbb{R}^{n+1}\right)$ for all $\zeta \in S_{\nu^{\prime}}\left(\mathbb{C}^{n}\right)$. To see this, suppose that $\zeta=\xi+i \eta \in S_{\nu^{\prime}}\left(\mathbb{C}^{n}\right)$ and that the cone $H_{\theta}^{+}$defined by formula (15) is tangential to one of the faces of $D_{\zeta, \delta_{\zeta}}$ normal to $\eta \neq 0$ and the other face is contained in $S_{\theta}\left(\mathbb{R}^{n+1}\right)$. According to equation (16), $\delta_{\zeta}>0$ satisfies one of the quadratic equations

$$
\left(1+\delta_{\zeta}\right)^{2}|\eta|^{2}=\sin ^{2} \theta \cdot\left(\left|\xi \pm \delta_{\zeta} \eta / 2\right|^{2}+\tan ^{2} \theta \cdot\left\langle\xi \pm \delta_{\zeta} \eta / 2, \widehat{\eta}\right\rangle^{2}\right)
$$

with $\widehat{\eta}=\eta /|\eta|$. Then $\delta=\inf \left\{\delta_{\zeta}: \zeta \in S_{\nu^{\prime}}\left(\mathbb{C}^{n}\right),|\zeta|=1\right\}$ is the required positive number because $\delta_{t \zeta}=\delta_{\zeta}$ for all $t>0$ and $\zeta \in S_{\nu^{\prime}}\left(\mathbb{C}^{n}\right)$ with $\eta \neq 0$. The infimum is attained when $|\xi| /|\eta|$ is bounded away from zero.

By Cauchy's theorem in Clifford analysis, we have

$$
\tilde{f}(\zeta)=\int_{\partial D_{\zeta, \delta}} G_{x}(\zeta) \boldsymbol{n}(x) f(x) d \mu(x) .
$$

Although the boundary $\partial D_{\zeta, \delta}$ is not smooth, the edges can be smoothed out to obtain the given representation. Then by Lemma 3.3,

$$
\begin{aligned}
|\widetilde{f}(\zeta)| & \leq 2^{n / 2}\|f\|_{\nu, \infty} \int_{\partial D_{\zeta, \delta}}\left|G_{x}(\zeta)\right| d \mu(x) \\
& \leq 3 \cdot 2^{n / 2}\|f\|_{\nu, \infty} \frac{(1+\delta / 2)(1+\delta)^{n}\left(2 \Sigma_{n}+\sigma_{n-1} \delta\right)}{\sigma_{n} \varepsilon_{\delta}^{n+1}}
\end{aligned}
$$

for all $\zeta \in S_{\nu^{\prime}}\left(\mathbb{C}^{n}\right)$. The positive numbers $\delta$ and $\varepsilon_{\delta}$ depend only on $n, \nu^{\prime}$ and $\nu^{\prime}<\theta<\nu$.

4. Bounded holomorphic functions in sectors. To complete the proof of Theorem 2.4, we show in this section that the mapping $f \mapsto$ 
$\widetilde{f}$ given by the Cauchy integral formula (13) maps $H_{\ell}^{\infty}\left(S_{\nu}^{\circ}\left(\mathbb{R}^{n+1}\right)\right)$ onto $H^{\infty}\left(S_{\nu}^{\circ}\left(\mathbb{C}^{n}\right)\right)$. To achieve this, we construct the inverse map and show that for every $0<\nu^{\prime}<\nu^{\prime \prime}<\nu$, there exists $C_{\nu^{\prime}, \nu^{\prime \prime}}>0$ such that for all $\tilde{f} \in$ $H^{\infty}\left(S_{\nu}^{\circ}\left(\mathbb{C}^{n}\right)\right)$, the supremum of $|\widetilde{f}|$ over the subsector $S_{\nu^{\prime}}^{\circ}\left(\mathbb{C}^{n}\right)$ is bounded by $C_{\nu^{\prime}, \nu^{\prime \prime}}$ times the supremum of $|f|$ over the subsector $S_{\nu^{\prime \prime}}^{\circ}\left(\mathbb{R}^{n+1}\right)$.

The second half of the proof of Theorem 2.4 has a different flavour to the proof of the first half given in the preceding section. Here we appeal to the Fourier theory of monogenic functions exposed in [8]. As mentioned in Section 2, the sector $S_{\nu}\left(\mathbb{C}^{n}\right)$ arose in [8] as the set of $\zeta \in \mathbb{C}^{n}$ for which the exponential functions (19) have decay at infinity for all $x \in \mathbb{R}^{n+1}$ with $\langle x, m\rangle>0$ and all unit vectors $m=m_{0} e_{0}+\boldsymbol{m} \in \mathbb{R}^{n+1}$ satisfying $m_{0} \geq$ $|\boldsymbol{m}| \cot \nu$. We exploit this property to construct a left monogenic function $f: S_{\nu}^{\circ}\left(\mathbb{R}^{n+1}\right) \rightarrow C \ell\left(\mathbb{C}^{n}\right)$ bounded on subsectors from a holomorphic function $\widetilde{f}: S_{\nu}^{\circ}\left(\mathbb{C}^{n}\right) \rightarrow C \ell\left(\mathbb{C}^{n}\right)$ bounded on subsectors. Before doing so, we recall some facts about the sectors $S_{\nu}^{\circ}\left(\mathbb{C}^{n}\right)$ from [8, Section 4].

4.1. Sectors in $\mathbb{C}^{n}$. For each unit vector $m \in \mathbb{R}^{n+1}$ with $m=m_{0} e_{0}+\boldsymbol{m}$ satisfying $m_{0}>0$, the real $n$-dimensional manifold $m\left(\mathbb{C}^{n}\right)$ in $\mathbb{C}^{n}$ is defined as the set of all nonzero $\zeta=\xi+i \eta \in \mathbb{C}^{n}$ such that $\xi, \eta \in \mathbb{R}^{n}$ satisfy equation (17), or equivalently, equation (18).

According to the proof of Proposition 2.1, the manifold $m\left(\mathbb{C}^{n}\right)$ is the collection of all $\zeta=\xi+i \eta \in \mathbb{C}^{n}$ such that $\eta \in \mathbb{R}^{n}$ lies in the direction of $\boldsymbol{m}$ and the monogenic spectrum $\gamma(\zeta)$ of $\zeta$ is tangential to the cone $H_{\theta}^{+}$ given by (15) with $\tan \theta=m_{0} /|\boldsymbol{m}|$. Manifolds associated with distinct unit vectors $m$ are disjoint. Moreover, for $0<\omega<\pi / 2$, the sector $S_{\omega}\left(\mathbb{C}^{n}\right)$ of all $\zeta \in \mathbb{C}^{n}$ satisfying condition (14) is the disjoint union of the manifolds $m\left(\mathbb{C}^{n}\right)$ for all unit vectors $m \in \mathbb{R}^{n+1}$ with $m=m_{0} e_{0}+\boldsymbol{m}$ and $m_{0} \geq|\boldsymbol{m}| \cot \omega$ and $\{0\}$. Its interior $S_{\omega}^{\circ}\left(\mathbb{C}^{n}\right)$ is the union of all such manifolds $m\left(\mathbb{C}^{n}\right)$ with $m_{0}>|\boldsymbol{m}| \cot \omega$. For the vector $m=e_{0}$, we have $e_{0}\left(\mathbb{C}^{n}\right)=\mathbb{R}^{n} \backslash\{0\}$.

Let $m \in \mathbb{R}^{n+1}$ be a unit vector with $m=m_{0} e_{0}+\boldsymbol{m}$ satisfying $m_{0}>0$. For all $\zeta=\xi+i \eta \in m\left(\mathbb{C}^{n}\right)$ with $\xi, \eta \in \mathbb{R}^{n}$, the quantities $|\xi|,|\zeta|, \Re\left(|\zeta|_{\mathbb{C}}\right)$ and $\left.|| \zeta\right|_{\mathbb{C}} \mid$ are equivalent:

$$
\Re|\zeta|_{\mathbb{C}} \leq|\xi| \leq \frac{\Re|\zeta|_{\mathbb{C}}}{m_{0}}
$$

and

$$
\Re|\zeta|_{\mathbb{C}} \leq\left.|| \zeta\right|_{\mathbb{C}}\left|\leq \frac{\Re|\zeta|_{\mathbb{C}}}{m_{0}} \leq\right| \zeta \mid \leq \frac{\sqrt{1+|\boldsymbol{m}|^{2}} \Re|\zeta|_{\mathbb{C}}}{m_{0}}, \quad \zeta \in m\left(\mathbb{C}^{n}\right) .
$$

The Jacobian $\operatorname{det}\left(\partial \zeta_{j} / \partial \xi_{k}\right)$ of the parametrisation $\xi \mapsto \xi+i \eta$ of $m\left(\mathbb{C}^{n}\right)$ given by formula (17) satisfies the bound

$$
\left|\operatorname{det}\left(\frac{\partial \zeta_{j}}{\partial \xi_{k}}\right)\right| \leq \frac{1}{m_{0}} .
$$


The pullback of a differential form $\omega$ on $\mathbb{C}^{n}$ via the embedding of $m\left(\mathbb{C}^{n}\right)$ in $\mathbb{C}^{n}$ is denoted by the same symbol. In particular, integration with respect to the complex $n$-form $d \zeta_{1} \wedge \cdots \wedge d \zeta_{n}$ on $m\left(\mathbb{C}^{n}\right)$ is equivalent to integration with respect to surface measure on $m\left(\mathbb{C}^{n}\right)$. The symbol $\left|d \zeta_{1} \wedge \cdots \wedge d \zeta_{n}\right|$ is used to denote the image of the measure $\left|\operatorname{det}\left(\partial \zeta_{j} / \partial \xi_{k}\right)\right| d \xi$ with respect to the parametrisation $\xi \mapsto \xi+i \eta$ of $m\left(\mathbb{C}^{n}\right)$, that is, the surface measure $d \mu$ of the $n$-dimensional real manifold $m\left(\mathbb{C}^{n}\right)$.

Besides the exponential function $e_{+}(x, \zeta)$ defined by formula (19), the function

$$
e_{-}(x, \zeta)=e^{i\langle x, \zeta\rangle} e^{x_{0}|\zeta|_{\mathbb{c}}} \chi_{-}(\zeta), \quad x=x_{0} e_{0}+\boldsymbol{x}, \zeta \in \mathbb{C}^{n},|\zeta|_{\mathbb{C}}^{2} \notin(-\infty, 0],
$$

is also important. The projection $\chi_{-}(\zeta)$ is defined by formula (8). Then the functions $(x, \zeta) \mapsto e_{ \pm}(x, \zeta)$ are left monogenic in $x \in \mathbb{R}^{n+1}$ and holomorphic in $\zeta \in \mathbb{C}^{n}$. Let $m \in \mathbb{R}^{n+1}$ be a unit vector with $m=m_{0} e_{0}+\boldsymbol{m}$ satisfying $m_{0} \geq|\boldsymbol{m}| \cot \nu$. Then for $x=x_{0} e_{0}+\boldsymbol{x} \in \mathbb{R}^{n+1}$ and $\zeta=\xi+i \eta \in \mathbb{C}^{n}$, the bounds

$$
\begin{array}{ll}
\left|e_{+}(x, \zeta)\right|=e^{-\langle x, \eta\rangle-x_{0} \Re|\zeta| \mathbb{c}}\left|\chi_{+}(\zeta)\right| \leq \frac{\sec \nu}{\sqrt{2}} e^{-\langle x, m\rangle \Re|\zeta| \mathbb{c} / m_{0}}, \quad \zeta \in m\left(\mathbb{C}^{n}\right), \\
\left|e_{-}(x, \zeta)\right|=e^{-\langle x, \eta\rangle+x_{0} \Re|\zeta| \mathbb{C}}\left|\chi_{-}(\zeta)\right| \leq \frac{\sec \nu}{\sqrt{2}} e^{\langle x, m\rangle \Re|\zeta| c / m_{0}}, \quad \zeta \in \bar{m}\left(\mathbb{C}^{n}\right),
\end{array}
$$

are valid.

The set of $x=\left(x_{0}, x_{1}, \ldots, x_{n}\right) \in \mathbb{R}^{n+1}$ with $x_{0}>0$ is written as $\mathbb{R}_{+}^{n+1}$ and for $x_{0}<0$, as $\mathbb{R}_{-}^{n+1}$. For $0<\nu<\pi / 2$, let

$$
\begin{aligned}
& C_{\nu}^{+}\left(\mathbb{R}^{n+1}\right)=\left\{x \in \mathbb{R}^{n+1}: x=x_{0} e_{0}+\boldsymbol{x}, x_{0}>-|\boldsymbol{x}| \tan \nu\right\}, \\
& C_{\nu}^{-}\left(\mathbb{R}^{n+1}\right)=\left\{x \in \mathbb{R}^{n+1}: x=x_{0} e_{0}+\boldsymbol{x}, x_{0}<|\boldsymbol{x}| \tan \nu\right\}=-C_{\nu}^{+}\left(\mathbb{R}^{n+1}\right) .
\end{aligned}
$$

Then $S_{\nu}^{\circ}\left(\mathbb{R}^{n+1}\right)=C_{\nu}^{+}\left(\mathbb{R}^{n+1}\right) \cap C_{\nu}^{-}\left(\mathbb{R}^{n+1}\right)$. Given a unit vector $m=m_{0} e_{0}+$ $\boldsymbol{m} \in \mathbb{R}^{n+1}$, let $H_{m}$ denote the half-space $\left\{x \in \mathbb{R}^{n+1}:\langle x, m\rangle>0\right\}$. We also note here that

$$
\begin{aligned}
& C_{\nu}^{+}\left(\mathbb{R}^{n+1}\right)=\bigcup\left\{H_{m}: m \in S^{n}, m=m_{0} e_{0}+\boldsymbol{m}, m_{0}>|\boldsymbol{m}| \cot \nu\right\}, \\
& C_{\nu}^{-}\left(\mathbb{R}^{n+1}\right)=\bigcup\left\{-H_{m}: m \in S^{n}, m=m_{0} e_{0}+\boldsymbol{m}, m_{0}>|\boldsymbol{m}| \cot \nu\right\} .
\end{aligned}
$$

4.2. Fourier analysis in sectors. For each $\zeta \in \mathbb{C}^{n}$ such that $|\zeta|_{\mathbb{C}}^{2} \notin$ $(-\infty, 0]$, set $\psi(\zeta)=\chi_{+}(\zeta)|\zeta|_{\mathbb{C}} e^{-|\zeta|_{\mathbb{C}}}$ and $\psi_{t}(\zeta)=\psi(t \zeta)$ for $t>0$. Suppose that $b: S_{\nu}^{\circ}\left(\mathbb{C}^{n}\right) \rightarrow C \ell\left(\mathbb{C}^{n}\right)$ is a uniformly bounded holomorphic function. Then for each $t>0$, the product $b \cdot \psi_{t}$ is a bounded holomorphic function with exponential decay at infinity in $S_{\nu}^{\circ}\left(\mathbb{C}^{n}\right)$. Hence, the Fourier transform

$$
\left(b \cdot \psi_{t}\right)^{\wedge}(\xi)=\int_{\mathbb{R}^{n}} e^{-i\langle x, \xi\rangle} b(x) \psi_{t}(x) d x
$$

converges for all $\xi \in \mathbb{R}^{n}$. 
Lemma 4.1. Let $b: S_{\nu}^{\circ}\left(\mathbb{C}^{n}\right) \rightarrow C \ell\left(\mathbb{C}^{n}\right)$ be a uniformly bounded holomorphic function. Then for each $t>0$, the Fourier transform $\left(b \cdot \psi_{t}\right)^{\wedge}: \mathbb{R}^{n} \rightarrow$ $C \ell\left(\mathbb{C}^{n}\right)$ has a left monogenic extension to $C_{\nu}^{-}\left(\mathbb{R}^{n+1}\right)$ (denoted by the same symbol). Moreover, for every $0<\nu^{\prime}<\nu$, there exists $B_{\nu^{\prime}}>0$ such that for every uniformly bounded holomorphic function $b: S_{\nu}^{\circ}\left(\mathbb{C}^{n}\right) \rightarrow C \ell\left(\mathbb{C}^{n}\right)$, the bound

$$
\left|\left(b \cdot \psi_{t}\right)^{\wedge}(x)\right| \leq B_{\nu^{\prime}}\|b\|_{\infty} \frac{|x| / t}{|x|^{n}\left(1+|x|^{2} / t^{2}\right)}, \quad x \in C_{\nu^{\prime}}^{-}\left(\mathbb{R}^{n+1}\right),
$$

holds for all $t>0$.

Proof. Let $m=m_{0} e_{0}+\boldsymbol{m} \in \mathbb{R}^{n+1}$ satisfy $m_{0}>|\boldsymbol{m}| \cot \nu$. Set

$$
f_{m}(x)=\int_{m\left(\mathbb{C}^{n}\right)} e_{+}(-x, \zeta) b(\zeta) \psi_{t}(\zeta) d \zeta_{1} \wedge \cdots \wedge d \zeta_{n}
$$

for all $x \in \mathbb{R}^{n+1}$ such that $\langle x, m\rangle<0$. Then $f_{m}$ is left monogenic in the set $\left\{x \in \mathbb{R}^{n+1}:\langle x, m\rangle<0\right\}$ because $e_{+}(-x, \zeta)$ is left monogenic in $x$ and has exponential decay in $\zeta$ for $\langle x, m\rangle<0$ according to the bound (25). By dominated convergence, $\lim _{x_{0} \rightarrow 0-} f_{e_{0}}\left(x_{0} e_{0}+\boldsymbol{x}\right)=\left(b \cdot \psi_{t}\right)^{\wedge}(\boldsymbol{x})$, so $f_{e_{0}}$ is the left monogenic extension of $\left(b \cdot \psi_{t}\right)^{\wedge}$ to $\mathbb{R}_{-}^{n+1}$.

For $x \in \mathbb{R}^{n+1}$ fixed, $m \mapsto f_{m}(x)$ is constant on the set of unit vectors $m=m_{0} e_{0}+\boldsymbol{m} \in \mathbb{R}^{n+1}$ satisfying $m_{0}>|\boldsymbol{m}| \cot \nu$ and $\langle x, m\rangle<0$ by Cauchy's theorem (see [10, p. 70]). It follows that $f_{m}$ is the unique extension of $\left(b \cdot \psi_{t}\right)^{\wedge}$ from $\mathbb{R}_{-}^{n+1}$ to all of $\left\{x \in \mathbb{R}^{n+1}:\langle x, m\rangle<0\right\}$. Because $C_{\nu}^{-}\left(\mathbb{R}^{n+1}\right)$ is the union of these sets for all unit vectors $m=m_{0} e_{0}+\boldsymbol{m} \in \mathbb{R}^{n+1}$ satisfying $m_{0}>|\boldsymbol{m}| \cot \nu$, the Fourier transform $\left(b \cdot \psi_{t}\right)^{\wedge}$ has a left monogenic extension to $C_{\nu}^{-}\left(\mathbb{R}^{n+1}\right)$ given by formula (28). Denote this left monogenic extension by $\left(b \cdot \psi_{t}\right)^{\wedge}$ as well.

To check the bound (27), we note that

$$
\begin{aligned}
\left|\left(b \cdot \psi_{t}\right)^{\wedge}(x)\right| & \leq \int_{m\left(\mathbb{C}^{n}\right)}\left|e_{+}(-x, \zeta)\right| \cdot\left|b(\zeta) \psi_{t}(\zeta)\right|\left|d \zeta_{1} \wedge \cdots \wedge d \zeta_{n}\right| \\
& \leq \frac{t\|b\|_{\infty}}{\sqrt{2} \cos \nu} \int_{m\left(\mathbb{C}^{n}\right)} e^{\langle x, m\rangle \Re|\zeta| \mathbb{C} / m_{0}}|| \zeta|\mathbb{C}| e^{-t \Re|\zeta| \mathbb{C}}\left|d \zeta_{1} \wedge \cdots \wedge d \zeta_{n}\right| \\
& \leq \frac{t\|b\|_{\infty}}{\sqrt{2} m_{0}^{2} \cos \nu} \int_{\mathbb{R}^{n}} e^{\left(\langle x, m\rangle-t m_{0}\right)|\xi|}|\xi| d \xi \\
& =\|b\|_{\infty} \frac{n ! \sigma_{n-1}}{\sqrt{2} m_{0}^{2} \cos \nu} \frac{t}{\left(-\langle x, m\rangle+t m_{0}\right)^{n+1}}
\end{aligned}
$$

for all $x \in C_{\nu}^{-}\left(\mathbb{R}^{n+1}\right)$ and all unit vectors $m=m_{0} e_{0}+\boldsymbol{m} \in \mathbb{R}^{n+1}$ satisfying $m_{0}>|\boldsymbol{m}| \cot \nu$ and $\langle x, m\rangle<0$. Here we have appealed to the bounds (21) and (22) on the manifolds $m\left(\mathbb{C}^{n}\right)$ and the bound (23) for the Jacobian of the parametrization $\xi \mapsto \xi+i \eta$ of $m\left(\mathbb{C}^{n}\right)$. 
Now let $0<\nu^{\prime}<\nu$. There exists $a_{\nu^{\prime}}>0$ such that for any $x \in C_{\nu^{\prime}}^{-}\left(\mathbb{R}^{n+1}\right)$, we can choose a unit vector $m=m_{0} e_{0}+\boldsymbol{m} \in \mathbb{R}^{n+1}$ satisfying $m_{0}>|\boldsymbol{m}| \cot \nu$ and $-\langle x, m\rangle \geq a_{\nu^{\prime}}|x|$ with $a_{\nu^{\prime}}$ independent of $x$ and $m$. Then

$$
\frac{t}{\left(a_{\nu^{\prime}}|x|+t m_{0}\right)^{n+1}} \leq \frac{A_{\nu^{\prime}}}{|x|^{n}} \frac{|x|^{n} / t^{n}}{(|x| / t+1)^{n+1}} \leq \frac{A_{\nu^{\prime}}}{|x|^{n}} \frac{|x| / t}{1+|x|^{2} / t^{2}}
$$

and we obtain the bound (27).

We also need some bounds on the denominator of the Cauchy kernel (9).

Proposition 4.2. (i) The bound

$$
|| x-\zeta|\mathbb{C}| \geq|x|\left(1-\kappa^{-1}\right)
$$

holds for all $\kappa \geq 1, x \in \mathbb{R}^{n+1}$ and $\zeta=\xi+i \eta \in \mathbb{C}^{n}$ with $|x| \geq$ $\kappa(|\xi|+|\eta|)$.

(ii) The bound

$$
|| x-\zeta|\mathbb{C}| \leq 2|x|
$$

holds for all $x \in \mathbb{R}^{n+1}$ with $|x| \geq|\zeta|$.

(iii) Let $0<\nu<\pi / 2$. The bound

$$
|| \zeta-\left.x\right|_{\mathbb{C}}\left|\geq \frac{\cos \nu}{\left(1+\sin ^{2} \nu\right)^{1 / 2}}\left(1-\kappa^{-1}\right)\right| \zeta \mid
$$

holds for all $\kappa>1, x \in \mathbb{R}^{n+1}$ and $\zeta \in S_{\nu}^{\circ}\left(\mathbb{C}^{n}\right)$ such that

$$
|\zeta| \geq \kappa\left((1+2 \sqrt{2}) \frac{1+\sin ^{2} \nu}{\cos ^{2} \nu}\right)|x| .
$$

(iv) For every $0<\nu<\theta<\pi / 2$, there exists $\varepsilon_{\nu, \theta}>0$ such that

$$
|| x-\zeta|\mathbb{C}|>\varepsilon_{\nu, \theta}|x|
$$

for all $\zeta \in S_{\nu}^{\circ}\left(\mathbb{C}^{n}\right)$ and $x \in \mathbb{R}^{n+1} \backslash S_{\theta}^{\circ}\left(\mathbb{R}^{n+1}\right)$.

(v) For every $0<\nu<\theta<\pi / 2$, there exists $\varepsilon_{\nu, \theta}^{\prime}>0$ such that

$$
|| x-\zeta|\mathbb{C}|>\varepsilon_{\nu, \theta}^{\prime}|\zeta|
$$

for all $\zeta \in S_{\nu}^{\circ}\left(\mathbb{C}^{n}\right)$ and $x \in \mathbb{R}^{n+1} \backslash S_{\theta}^{\circ}\left(\mathbb{R}^{n+1}\right)$.

Proof. (i) Let $x=x_{0} e_{0}+\boldsymbol{x} \in \mathbb{R}^{n+1}$ and $\zeta=\xi+i \eta \in \mathbb{C}^{n}$. Then

$$
\begin{aligned}
|| x-\left.\left.\zeta\right|_{\mathbb{C}} ^{2}\right|^{2} & =\left|x_{0}^{2}+\right| \boldsymbol{x}-\left.\xi\right|^{2}-|\eta|^{2}-\left.2 i\langle\boldsymbol{x}-\xi, \eta\rangle\right|^{2} \\
& =\left(x_{0}^{2}+|\boldsymbol{x}-\xi|^{2}-|\eta|^{2}\right)^{2}+4\langle\boldsymbol{x}-\xi, \eta\rangle^{2} \\
& \geq\left(x_{0}^{2}+|\boldsymbol{x}-\xi|^{2}-|\eta|^{2}\right)^{2}=|x|^{4}\left(\left.|x /| x|-\xi /| x\right|^{2}-|\eta|^{2} /|x|^{2}\right)^{2},
\end{aligned}
$$

where we have identified $\mathbb{R}^{n}$ with the subspace $\{0\} \times \mathbb{R}^{n}$ of $\mathbb{R}^{n+1}$. Now $|x /| x|-\xi /| x|| \geq 1-|\xi| /|x|$ for $|x| \geq|\xi|$, so in this case, we have

$$
\begin{aligned}
|| x-\left.\left.\zeta\right|_{\mathbb{C}} ^{2}\right|^{2} & \geq|x|^{4}\left((1-|\xi| /|x|)^{2}-|\eta|^{2} /|x|^{2}\right)^{2} \\
& =|x|^{4}\left(1-\frac{|\xi|+|\eta|}{|x|}\right)^{2}\left(1-\frac{|\xi|-|\eta|}{|x|}\right)^{2} \geq|x|^{4}\left(1-\frac{|\xi|+|\eta|}{|x|}\right)^{4} .
\end{aligned}
$$


Hence, ||$x-\left.\zeta\right|_{\mathbb{C}}|\geq| x \mid\left(1-\kappa^{-1}\right)$ for all $x \in \mathbb{R}^{n+1}$ with $|x| \geq \kappa(|\xi|+|\eta|)$ and all $\kappa \geq 1$.

(ii) On the other hand, if $x \in \mathbb{R}^{n+1}, \zeta=\xi+i \eta \in \mathbb{C}^{n}$ and $|x| \geq \beta\left(|\xi|^{2}+\right.$ $\left.|\eta|^{2}\right)^{1 / 2}$ for $\beta \geq 1$, then

$$
\begin{aligned}
|| x-\left.\left.\zeta\right|_{\mathbb{C}} ^{2}\right|^{2} & \leq|x|^{4}\left(|x /| x|-\xi /| x||^{2}-|\eta|^{2} /|x|^{2}\right)^{2}+4|x-\xi|^{2}|\eta|^{2} \\
& \leq|x|^{4}\left((1+|\xi| /|x|)^{2}+|\eta|^{2} /|x|^{2}\right)^{2} \\
& \leq|x|^{4}\left(1+\frac{2|\xi|}{|x|}+\frac{1}{\beta^{2}}\right)^{2} \leq 2^{4}|x|^{4}
\end{aligned}
$$

Hence, ||$x-\zeta|\mathbb{C}| \leq 2|x|$ for all $x \in \mathbb{R}^{n+1}$ with $|x| \geq\left(|\xi|^{2}+|\eta|^{2}\right)^{1 / 2}=|\zeta|$.

(iii) Here we are looking at the limiting behaviour of $|\zeta-x|_{\mathbb{C}}$ as $|\zeta| \rightarrow \infty$ in $S_{\nu}^{\circ}\left(\mathbb{C}^{n}\right)$. Let $m=m_{0} e_{0}+\boldsymbol{m}$ be a unit vector in $\mathbb{R}^{n+1}$ such that $m_{0}>|\boldsymbol{m}| \cot \nu$ and let $m\left(\mathbb{C}^{n}\right)$ be the real manifold defined by equation (17) in $\mathbb{C}^{n}$. Then as noted above, $S_{\nu}^{\circ}\left(\mathbb{C}^{n}\right)$ is the union of all such manifolds, and ||$\zeta|\mathbb{C}|$ and $|\zeta|$ are comparable on $m\left(\mathbb{C}^{n}\right)$. Note that by continuity,

$$
|| \zeta /|\zeta|-x /|\zeta|_{\mathbb{C}} \mid \approx \frac{\left.|| \zeta\right|_{\mathbb{C}} \mid}{|\zeta|} \geq \frac{m_{0}}{\left(1+|\boldsymbol{m}|^{2}\right)^{1 / 2}}
$$

on $m\left(\mathbb{C}^{n}\right)$ as $|\zeta| \rightarrow \infty$. Indeed, suppose that $\zeta \in m\left(\mathbb{C}^{n}\right)$ and $|\zeta|=1$. Then

$$
\begin{aligned}
|| \zeta-\left.x\right|_{\mathbb{C}}-|\zeta|_{\mathbb{C}} \mid & =\frac{|| \zeta-\left.x\right|_{\mathbb{C}} ^{2}-|\zeta|_{\mathbb{C}}^{2} \mid}{|| \zeta-\left.x\right|_{\mathbb{C}}+|\zeta|_{\mathbb{C}} \mid} \leq \frac{|| \zeta-\left.x\right|_{\mathbb{C}} ^{2}-|\zeta|_{\mathbb{C}}^{2} \mid}{\Re|\zeta|_{\mathbb{C}}} \\
& \leq \frac{\left(1+|\boldsymbol{m}|^{2}\right)^{1 / 2}}{m_{0}}|| \zeta-\left.x\right|_{\mathbb{C}} ^{2}-|\zeta|_{\mathbb{C}}^{2} \mid
\end{aligned}
$$

by the estimates (22). If $x=x_{0} e_{0}+\boldsymbol{x}$ satisfies $|x| \leq 1$ and $\zeta=\xi+i \eta \in \mathbb{C}^{n}$ has norm one, we get

$$
\begin{aligned}
|| \zeta-\left.x\right|_{\mathbb{C}} ^{2}-|\zeta|_{\mathbb{C}}^{2} \mid & =\left|x_{0}^{2}+\right| \boldsymbol{x}-\left.\xi\right|^{2}-|\xi|^{2}-2 i\langle\eta, \boldsymbol{x}\rangle \mid \\
& \leq x_{0}^{2}+|| \xi-\boldsymbol{x}|-| \xi|| \cdot|| \xi-\boldsymbol{x}|+| \xi||+2|\eta| \cdot|\boldsymbol{x}| \\
& \leq x_{0}^{2}+|\boldsymbol{x}| \cdot(2|\xi|+|\boldsymbol{x}|)+2|\eta||\boldsymbol{x}| \\
& \leq x_{0}^{2}+|\boldsymbol{x}|^{2}+2(|\xi|+|\eta|)|\boldsymbol{x}| \\
& \leq(1+2|\xi|+2|\eta|)|x| \leq(1+2 \sqrt{2})|x|,
\end{aligned}
$$

so that ||$\zeta-\left.x\right|_{\mathbb{C}}-|\zeta|_{\mathbb{C}}\left|\leq(1+2 \sqrt{2})\left(1+|\boldsymbol{m}|^{2}\right)^{1 / 2}\right| x \mid / m_{0}$.

Hence, for all nonzero $\zeta \in m\left(\mathbb{C}^{n}\right)$ and $x \in \mathbb{R}^{n+1}$ satisfying $|x| \leq|\zeta|$, we have

$$
\begin{aligned}
|| \zeta /|\zeta|-x /|\zeta|_{\mathbb{C}} \mid & \geq \frac{\left.|| \zeta\right|_{\mathbb{C}} \mid}{|\zeta|}-(1+2 \sqrt{2}) \frac{\left(1+|\boldsymbol{m}|^{2}\right)^{1 / 2}}{m_{0}} \frac{|x|}{|\zeta|} \\
& \geq \frac{m_{0}}{\left(1+|\boldsymbol{m}|^{2}\right)^{1 / 2}}\left(1-(1+2 \sqrt{2}) \frac{1+|\boldsymbol{m}|^{2}}{m_{0}^{2}} \frac{|x|}{|\zeta|}\right)
\end{aligned}
$$


on appealing to the estimates (22) again. Now $|\boldsymbol{m}|^{2}\left(1+\cot ^{2} \nu\right) \leq 1=m_{0}^{2}+$ $|\boldsymbol{m}|^{2} \leq m_{0}^{2}\left(1+\tan ^{2} \nu\right)$, so $m_{0}>\cos \nu$ and $|\boldsymbol{m}|<\sin \nu$ and the inequality (iii) follows.

(iv) Let $0<\nu<\theta<\pi / 2$. Then there exists $\varepsilon_{\nu, \theta}>0$ such that

$$
|| x-\left.\zeta\right|_{\mathbb{C}} \mid>\varepsilon_{\nu, \theta}
$$

for all $\zeta \in S_{\nu}^{\circ}\left(\mathbb{C}^{n}\right)$ and unit vectors $x \in \mathbb{R}^{n+1} \backslash S_{\theta}^{\circ}\left(\mathbb{R}^{n+1}\right)$. To see this, let $m=m_{0} e_{0}+\boldsymbol{m}$ be a unit vector in $\mathbb{R}^{n+1}$ such that $m_{0}>|\boldsymbol{m}| \cot \nu$ and suppose that $\kappa>1$ and $\zeta \in m\left(\mathbb{C}^{n}\right)$ satisfies $|\zeta|>r=\kappa(1+2 \sqrt{2})\left(1+\sin ^{2} \nu\right) / \cos ^{2} \nu$. Then by (iii), we have

$$
|| x-\zeta|\mathbb{C}|>\frac{\cos \nu}{\left(1+\sin ^{2} \nu\right)^{1 / 2}}\left(1-\kappa^{-1}\right)|\zeta|>\frac{r \cos \nu}{\left(1+\sin ^{2} \nu\right)^{1 / 2}}\left(1-\kappa^{-1}\right) .
$$

On the other hand, according to Proposition 2.1, the function $(\zeta, x) \mapsto$ ||$x-\left.\zeta\right|_{\mathbb{C}} \mid$ is positive and continuous on the compact set

$$
\left(\{|\zeta| \leq r\} \cap S_{\nu}\left(\mathbb{C}^{n}\right)\right) \times\left(S^{n} \cap\left(\mathbb{R}^{n+1} \backslash S_{\theta}^{\circ}\left(\mathbb{R}^{n+1}\right)\right)\right),
$$

so it must be bounded below there. Then $\varepsilon_{\nu, \theta}$ is the minimum of these two lower bounds. It follows that ||$x-\left.\zeta\right|_{\mathbb{C}}\left|>\varepsilon_{\nu, \theta}\right| x \mid$ for all $\zeta \in S_{\nu}^{\circ}\left(\mathbb{C}^{n}\right)$ and $x \in \mathbb{R}^{n+1} \backslash S_{\theta}^{\circ}\left(\mathbb{R}^{n+1}\right)$.

(v) It suffices to prove the result for $|\zeta|=1$. According to (i), if $\kappa>1$ and $|x|>\sqrt{2} \kappa$, then ||$x-\left.\zeta\right|_{\mathbb{C}}|>| x \mid\left(1-\kappa^{-1}\right)>\sqrt{2}(\kappa-1)$ for all $\zeta \in \mathbb{C}^{n}$ with $|\zeta|=1$. On the other hand the function $(\zeta, x) \mapsto|| x-\left.\zeta\right|_{\mathbb{C}} \mid$ is positive and continuous on the compact set

$$
\left(\{|\zeta|=1\} \cap S_{\nu}\left(\mathbb{C}^{n}\right)\right) \times\left(\{|x| \leq \sqrt{2} \kappa\} \cap\left(\mathbb{R}^{n+1} \backslash S_{\theta}^{\circ}\left(\mathbb{R}^{n+1}\right)\right),\right.
$$

so it must be bounded below there. Then $\varepsilon_{\nu, \theta}^{\prime}$ is the minimum of these two lower bounds.

Lemma 4.3. The function $(x, y) \mapsto \widehat{\psi}_{t}(x-y), x, y \in \mathbb{R}^{n}, t>0$, is the restriction to $\mathbb{R}^{n} \times \mathbb{R}^{n}$ of a function $(\zeta, y) \mapsto \widehat{\psi}_{t}(\zeta-y)$ which is holomorphic in $\zeta \in \mathbb{C}^{n}$ and two-sided monogenic for all $y \in \mathbb{R}^{n+1}$ with $y+t e_{0} \notin \gamma(\zeta)$. Moreover,

$$
\widehat{\psi}_{t}(\zeta-y)=-\frac{(2 \pi)^{n}}{\sigma_{n}}\left(\frac{t e_{0}}{\left|y-\zeta+t e_{0}\right|_{\mathbb{C}}^{n+1}}-(n+1) \frac{\left(\bar{y}+\zeta+t e_{0}\right) t^{2}}{\left|y-\zeta+t e_{0}\right|_{\mathbb{C}}^{n+3}}\right) .
$$

Proof. We first note that that the Cauchy kernel (4) can be represented by

$$
k\left(x+t e_{0}\right)=\frac{1}{(2 \pi)^{n}} \int_{\mathbb{R}^{n}} e_{+}(x, \xi) \chi_{+}(\xi) e^{-t|\xi|} d \xi
$$

for all $x \in \mathbb{R}_{+}^{n+1}$ and $t>0$, so

$$
\widehat{\psi}_{t}(x-y)=\int_{\mathbb{R}^{n}} e_{+}(-(x-y), \xi) \psi_{t}(\xi) d \xi=-(2 \pi)^{n} t \frac{\partial}{\partial t} k\left(y-x+t e_{0}\right)
$$


for all $x, y \in \mathbb{R}^{n}$ and $t>0$. Holomorphically extending in $x$ and monogenically extending in $y$ gives the expression (29), defined for all $y \in \mathbb{R}^{n+1}$ and $\zeta \in \mathbb{C}^{n}$ with $y+t e_{0} \notin \gamma(\zeta)$.

Lemma 4.4. Let $b: S_{\nu}^{\circ}\left(\mathbb{C}^{n}\right) \rightarrow C \ell\left(\mathbb{C}^{n}\right)$ be a uniformly bounded holomorphic function. Then for each $t>0$ and $0<\nu^{\prime}<\nu$, the Fourier transform $\left(b \cdot \psi_{t}^{2}\right)^{\wedge}$ has a holomorphic extension to $S_{\nu^{\prime}}^{\circ}\left(\mathbb{C}^{n}\right)$ (denoted by the same symbol) given by

$$
\left(b \cdot \psi_{t}^{2}\right)^{\wedge}(\zeta)=\frac{1}{(2 \pi)^{n}} \int_{G_{\theta}} \widehat{\psi}_{t}(\zeta-y) \boldsymbol{n}(y)\left(b \cdot \psi_{t}\right)^{\wedge}(y) d \mu(y), \quad \zeta \in S_{\nu^{\prime}}^{\circ}\left(\mathbb{C}^{n}\right),
$$

where $\nu^{\prime}<\theta<\nu$ and

$$
G_{\theta}=\left\{y \in \mathbb{R}^{n+1}: y=y_{0} e_{0}+\boldsymbol{y}, y_{0}=|\boldsymbol{y}| \tan \theta\right\} .
$$

Proof. First, suppose that $\zeta=x \in \mathbb{R}^{n}$. Then $y \mapsto \widehat{\psi}_{t}(x-y)$ is uniformly bounded and two-sided monogenic in $\mathbb{R}_{+}^{n+1}$ and $\left(b \cdot \psi_{t}\right)^{\wedge}$ is left monogenic in $C_{\nu}^{-}\left(\mathbb{R}^{n+1}\right)$ by Lemma 4.1 .

According to the bound (27) we have

$$
\int_{G_{\theta}}\left|\left(b \cdot \psi_{t}\right)^{\wedge}(y)\right| d \mu(y) \leq C\|b\|_{\infty} \int_{0}^{\infty} \frac{r / t}{\left(1+\sec ^{2} \theta \cdot r^{2} / t^{2}\right)} \frac{d r}{r}<\infty,
$$

so that the integral (30) converges for all $0 \leq \theta<\nu$. The convolution formula

$$
\left(b \cdot \psi_{t}^{2}\right)^{\wedge}(x)=\frac{1}{(2 \pi)^{n}}\left(\widehat{\psi}_{t} *(b \cdot \psi)_{t}^{\wedge}\right)(x), \quad x \in \mathbb{R}^{n},
$$

and Cauchy's theorem in Clifford analysis now gives the representation (30). Then we can holomorphically extend the integral and the equality (30) for all $\zeta$ in $S_{\nu^{\prime}}^{\circ}\left(\mathbb{C}^{n}\right)$.

We need to check that for $\zeta$ in a fixed compact subset of $S_{\nu^{\prime}}^{\circ}\left(\mathbb{C}^{n}\right)$, the function $y \mapsto \widehat{\psi}_{t}(\zeta-y)$ is uniformly bounded for all $y \in G_{\theta}$. For $y \in G_{\theta}$ and $|y|$ large, this follows from formula (29) and the estimate in Proposition 4.2(iv). For $|y|$ small, we note that for each $t>0$, the positive continuous function $(y, \zeta) \mapsto\left|y-\zeta+t e_{0}\right|_{\mathbb{C}}$ is necessarily bounded below on compact subsets of $G_{\theta} \times S_{\nu^{\prime}}^{\circ}\left(\mathbb{C}^{n}\right)$.

Lemma 4.5. Let $b: S_{\nu}^{\circ}\left(\mathbb{C}^{n}\right) \rightarrow C \ell\left(\mathbb{C}^{n}\right)$ be a uniformly bounded holomorphic function. Then for each $t>0$ and $0<\nu^{\prime}<\nu$, the restriction of $b \cdot \psi_{t}^{2}$ to $\mathbb{R}^{n}$ has a left monogenic extension $b \cdot \ell \psi_{t}^{2}$ to $S_{\nu^{\prime}}^{\circ}\left(\mathbb{R}^{n+1}\right)$. Moreover,

$$
\left|\left(b \cdot \ell \psi_{t}^{2}\right)(x)\right|= \begin{cases}O\left((t|x|)^{1 / 2}\right) & \text { as } t|x| \rightarrow 0 \text { in } S_{\nu^{\prime}}^{\circ}\left(\mathbb{R}^{n+1}\right), \\ O\left((t|x|)^{-n}\right) & \text { as } t|x| \rightarrow \infty \text { in } S_{\nu^{\prime}}^{\circ}\left(\mathbb{R}^{n+1}\right) .\end{cases}
$$

The order of convergence is uniform for $\|b\|_{\infty} \leq 1$. 
Proof. For each $x \in S_{\nu^{\prime}}^{\circ}\left(\mathbb{R}^{n+1}\right)=C_{\nu^{\prime}}^{+}\left(\mathbb{R}^{n+1}\right) \cap C_{\nu^{\prime}}^{-}\left(\mathbb{R}^{n+1}\right)$ set

$$
\begin{aligned}
& \left(b \cdot \ell \psi_{t}^{2}\right)_{+}(x)=\frac{1}{(2 \pi)^{n}} \int_{m\left(\mathbb{C}^{n}\right)} e_{+}(x, \zeta)\left(b \cdot \psi_{t}^{2}\right)^{\wedge}(\zeta) d \zeta_{1} \wedge \cdots \wedge d \zeta_{n}, \\
& \left(b \cdot \ell \psi_{t}^{2}\right)_{-}(x)=\frac{1}{(2 \pi)^{n}} \int_{m^{\prime}\left(\mathbb{C}^{n}\right)} e_{-}(x, \zeta)\left(b \cdot \psi_{t}^{2}\right)^{\wedge}(\zeta) d \zeta_{1} \wedge \cdots \wedge d \zeta_{n} \\
& \left(b \cdot \ell \psi_{t}^{2}\right)=\left(b \cdot \ell \psi_{t}^{2}\right)_{+}+\left(b \cdot \ell \psi_{t}^{2}\right)_{-} .
\end{aligned}
$$

Here $m=m_{0} e_{0}+\boldsymbol{m} \in \mathbb{R}^{n+1}$ and $m^{\prime}=m_{0}^{\prime} e_{0}+\boldsymbol{m}^{\prime} \in \mathbb{R}^{n+1}$ are two unit vectors satisfying $m_{0}>|\boldsymbol{m}| \cot \nu^{\prime}$ and $m_{0}^{\prime}>\left|\boldsymbol{m}^{\prime}\right| \cot \nu^{\prime}$ and $\langle x, m\rangle>0$ and $\left\langle x, m^{\prime}\right\rangle<0$. As mentioned in Section 4.1 above, the sector $S_{\nu^{\prime}}^{\circ}\left(\mathbb{C}^{n}\right)$ is the disjoint union of all manifolds $l\left(\mathbb{C}^{n}\right)$ for all unit vectors $l \in \mathbb{R}^{n+1}$ satisfying $l_{0}>|\boldsymbol{l}| \cot \nu^{\prime}$. According to Lemma 4.4, $\left(b \cdot \psi_{t}^{2}\right)^{\wedge}$ is defined on $S_{\nu^{\prime}}^{\circ}\left(\mathbb{C}^{n}\right)$. Once we establish the absolute convergence of the integrals (33) and (34), the argument of $[10$, p. 70$]$ shows that the right hand sides of equations (33) and (34) are independent of the choice of the unit vectors $m, m^{\prime}$, so that $\left(b \cdot \ell \psi_{t}^{2}\right)_{ \pm}$ and, hence, $\left(b \cdot \ell \psi_{t}^{2}\right)$ are well defined functions on $S_{\nu^{\prime}}^{\circ}\left(\mathbb{R}^{n+1}\right)$. Because the functions $e_{ \pm}(\cdot, \zeta)$ are left monogenic for each $\zeta \in \mathbb{C}^{n}$, the functions $\left(b \cdot \ell \psi_{t}^{2}\right)_{ \pm}$ and $\left(b \cdot \ell \psi_{t}^{2}\right)$ are left monogenic functions defined on $S_{\nu^{\prime}}^{\circ}\left(\mathbb{R}^{n+1}\right)$.

We first see that the right hand side of equation (33) converges for all $x \in C_{\nu^{\prime}}^{+}\left(\mathbb{R}^{n+1}\right)$. The integral is estimated for $0<\nu^{\prime}<\theta<\nu$ and $x \in C_{\nu^{\prime}}^{+}\left(\mathbb{R}^{n+1}\right)$ by

$$
\begin{aligned}
\left|\left(b \cdot \ell \psi_{t}^{2}\right)_{+}(x)\right| \leq & \frac{1}{(2 \pi)^{n}} \int_{m\left(\mathbb{C}^{n}\right)}\left|e_{+}(x, \zeta)\right| \cdot\left|\left(b \cdot \psi_{t}^{2}\right)^{\wedge}(\zeta)\right|\left|d \zeta_{1} \wedge \cdots \wedge d \zeta_{n}\right| \\
\leq & \frac{1}{(2 \pi)^{2 n}} \int_{m\left(\mathbb{C}^{n}\right)} \int_{G_{\theta}}\left|e_{+}(x, \zeta)\right| \cdot\left|\widehat{\psi}_{t}(\zeta-y)\right| \\
& \times\left|\left(b \cdot \psi_{t}\right)^{\wedge}(y)\right| d \mu(y)\left|d \zeta_{1} \wedge \cdots \wedge d \zeta_{n}\right| \\
= & \frac{1}{(2 \pi)^{2 n}} \int_{m\left(\mathbb{C}^{n}\right) G_{\theta}}\left|e_{+}(x, \zeta)\right| \cdot\left|\widehat{\psi}_{t /|\zeta|}(\zeta /|\zeta|-y /|\zeta|)\right| \\
& \times\left|\left(b \cdot \psi_{t}\right)^{\wedge}(y)\right| d \mu(y) \frac{\left|d \zeta_{1} \wedge \cdots \wedge d \zeta_{n}\right|}{|\zeta|^{n}} \\
= & \frac{1}{(2 \pi)^{2 n}} \int_{m\left(\mathbb{C}^{n}\right)} \int_{G_{\theta}}\left|e_{+}(t x, \zeta)\right| \cdot\left|\widehat{\psi}_{1 /|\zeta|}(\zeta /|\zeta|-y /(t|\zeta|))\right| \\
& \times\left|\left(b \cdot \psi_{t}\right)^{\wedge}(y)\right| d \mu(y) \frac{\left|d \zeta_{1} \wedge \cdots \wedge d \zeta_{n}\right|}{|\zeta|^{n}} \\
\leq & C_{\nu}^{\prime}\|b\|_{\infty} \int_{m\left(\mathbb{C}^{n}\right) G_{\theta}} \int_{G_{\theta}}\left|e_{+}(t x, \zeta)\right| \cdot\left|\widehat{\psi}_{1 /|\zeta|}(\zeta /|\zeta|-y /|\zeta|)\right| \\
& \times \frac{|y|}{1+|y|^{2}} \frac{d \mu(y)}{|y|^{n}} \frac{\left|d \zeta_{1} \wedge \cdots \wedge d \zeta_{n}\right|}{|\zeta|^{n}}
\end{aligned}
$$


Here we have used the explicit formula (29), the estimate (27) and the fact that both measures

$$
\frac{\left|d \zeta_{1} \wedge \cdots \wedge d \zeta_{n}\right|}{|\zeta|^{n}}, \quad \frac{d \mu(y)}{|y|^{n}}
$$

are invariant under dilations. It remains to estimate the function

$$
(y, \zeta) \mapsto\left|\widehat{\psi}_{1 /|\zeta|}(\zeta /|\zeta|-y /|\zeta|)\right|, \quad y \in G_{\theta}, \zeta \in m\left(\mathbb{C}^{n}\right),
$$

which is independent of $t>0$.

We now show that $\left|\widehat{\psi}_{1 /|\zeta|}(\zeta /|\zeta|-y /|\zeta|)\right|=O\left(|\zeta|^{-1}\right)$ uniformly in $y \in G_{\theta}$ as $|\zeta| \rightarrow \infty$ for $\zeta \in S_{\nu^{\prime}}\left(\mathbb{C}^{n}\right)$, and $\left|\widehat{\psi}_{1 /|\zeta|}(\zeta /|\zeta|-y /|\zeta|)\right|=O\left(|\zeta|^{n}\right)$ uniformly in $y \in G_{\theta}$ as $|\zeta| \rightarrow 0$ for $\zeta \in S_{\nu^{\prime}}\left(\mathbb{C}^{n}\right)$.

Let $\kappa>1$. Then by Proposition 4.2(i), for $\zeta^{\prime} \in m\left(\mathbb{C}^{n}\right)$ with $\left|\zeta^{\prime}\right|=1$ and all $x \in \mathbb{R}^{n+1}$ with $|x|>\sqrt{2} \kappa$, we have ||$x-\zeta^{\prime}|\mathbb{C}|>|x|\left(1-\kappa^{-1}\right)$. Set $x=\left(y+e_{0}\right) /|\zeta|$ for $y \in G_{\theta}$ and $\zeta \in m\left(\mathbb{C}^{n}\right)$. Comparison with formula (29) shows that

$$
\left|\widehat{\psi}_{1 /|\zeta|}(\zeta /|\zeta|-y /|\zeta|)\right| \leq \frac{(2 \pi)^{n}}{\sigma_{n} 2^{(n+1) / 2}|\zeta|}\left(\frac{1}{(\kappa-1)^{n+1}}+\frac{\sqrt{2} \kappa(n+1) /|\zeta|}{(\kappa-1)^{n+3}}\right)
$$
if $|x|>\sqrt{2} \kappa$.

According to Proposition $4.2(\mathrm{v})$, there exists $\varepsilon_{\nu, \theta}^{\prime}$ such that $\left|x-\zeta^{\prime}\right| \mathbb{C}>$ $\varepsilon_{\nu^{\prime}, \theta}^{\prime}$ for all $\zeta^{\prime} \in m\left(\mathbb{C}^{n}\right)$ with $\left|\zeta^{\prime}\right|=1$ and all $x \in \mathbb{R}^{n+1} \backslash S_{\theta}^{\circ}\left(\mathbb{R}^{n+1}\right)$, so if $x=\left(y+e_{0}\right) /|\zeta|$ for $y \in G_{\theta}$ and $|x| \leq \sqrt{2} \kappa$, we have

$$
\left|\widehat{\psi}_{1 /|\zeta|}(\zeta /|\zeta|-y /|\zeta|)\right| \leq \frac{(2 \pi)^{n}}{\sigma_{n}|\zeta|}\left(\frac{1}{\left(\varepsilon_{\nu^{\prime}, \theta}^{\prime}\right)^{n+1}}+(n+1) \frac{(\sqrt{2} \kappa+1) /|\zeta|}{\left(\varepsilon_{\nu^{\prime}, \theta}^{\prime}\right)^{n+3}}\right) .
$$

Hence, there exists $C>0$ such that

$$
\left|\widehat{\psi}_{1 /|\zeta|}(\zeta /|\zeta|-y /|\zeta|)\right| \leq \frac{C}{|\zeta|}
$$

for all $y \in G_{\theta}$ and $\zeta \in m\left(\mathbb{C}^{n}\right)$ with $|\zeta| \geq 1$.

On the other hand, $\widehat{\psi}_{1 /|\zeta|}(\zeta /|\zeta|-y /|\zeta|)=|\zeta|^{n} \widehat{\psi}_{1}(\zeta-y)$. Let $x=y+e_{0}$. According to Proposition 4.2(iv), there exists $\varepsilon_{\nu, \theta}$ such that $|x-\zeta|_{\mathbb{C}}>\varepsilon_{\nu^{\prime}, \theta}$ for all $\zeta \in m\left(\mathbb{C}^{n}\right)$. For $a>0$ and $x \in G_{\theta}$ such that $|x| \leq a$, we have

$$
\left|\widehat{\psi}_{1}(\zeta-y)\right| \leq \frac{(2 \pi)^{n}}{\sigma_{n}}\left(\frac{e_{0}}{\left(\varepsilon_{\nu^{\prime}, \theta}\right)^{n+1}}+(n+1) \frac{1+a}{\left(\varepsilon_{\nu^{\prime}, \theta}\right)^{n+3}}\right)
$$

for all $\zeta \in\{|\zeta| \leq 1\} \cap m\left(\mathbb{C}^{n}\right)$. Now let $\kappa>1$ and suppose that $a=\sqrt{2} \kappa$. Then by Proposition $4.2(\mathrm{i})$, for $\zeta \in m\left(\mathbb{C}^{n}\right)$ with $|\zeta| \leq 1$ and all $x \in \mathbb{R}^{n+1}$ with $|x|>\sqrt{2} \kappa$, we have ||$x-\zeta^{\prime}|\mathbb{C}|>|x|\left(1-\kappa^{-1}\right)$, so that

$$
\left|\widehat{\psi}_{1}(\zeta-y)\right| \leq \frac{(2 \pi)^{n}}{\sigma_{n} 2^{(n+1) / 2}}\left(\frac{e_{0}}{(\kappa-1)^{n+1}}+\frac{\sqrt{2} \kappa(n+1)}{(\kappa-1)^{n+3}}\right) .
$$


We have shown that $\left|\widehat{\psi}_{1 /|\zeta|}(\zeta /|\zeta|-y /|\zeta|)\right|=O\left(|\zeta|^{-1}\right)$ uniformly in $y \in G_{\theta}$ as $|\zeta| \rightarrow \infty$ for $\zeta \in S_{\nu^{\prime}}\left(\mathbb{C}^{n}\right)$, and $\left|\widehat{\psi}_{1 /|\zeta|}(\zeta /|\zeta|-y /|\zeta|)\right|=O\left(|\zeta|^{n}\right)$ uniformly in $y \in G_{\theta}$ as $|\zeta| \rightarrow 0$ for $\zeta \in S_{\nu^{\prime}}\left(\mathbb{C}^{n}\right)$. In particular,

$$
\int_{m\left(\mathbb{C}^{n}\right)} \sup _{y \in G_{\theta}}\left|\widehat{\psi}_{1 /|\zeta|}(\zeta /|\zeta|-y /|\zeta|)\right| \frac{\left|d \zeta_{1} \wedge \cdots \wedge d \zeta_{n}\right|}{|\zeta|^{n}}<\infty .
$$

Therefore,

$$
\begin{aligned}
\left|\left(b \cdot \ell \psi_{t}^{2}\right)_{+}(x)\right| \leq & C_{\nu}^{\prime \prime}\|b\|_{\infty} \int_{m\left(\mathbb{C}^{n}\right)}\left|e_{+}(t x, \zeta)\right| \\
& \times \sup _{y \in G_{\theta}}\left|\widehat{\psi}_{1 /|\zeta|}(\zeta /|\zeta|-y /|\zeta|)\right| \frac{\left|d \zeta_{1} \wedge \cdots \wedge d \zeta_{n}\right|}{|\zeta|^{n}} \\
\leq & C_{\nu}^{\prime \prime}\|b\|_{\infty} \sup _{y \in G_{\theta}, \zeta \in m\left(\mathbb{C}^{n}\right)}\left\{|\zeta|^{-n}\left|\widehat{\psi}_{1 /|\zeta|}(\zeta /|\zeta|-y /|\zeta|)\right|\right\} \\
& \times \int_{m\left(\mathbb{C}^{n}\right)}\left|e_{+}(t x, \zeta)\right|\left|d \zeta_{1} \wedge \cdots \wedge d \zeta_{n}\right| \\
\leq & C_{\nu}^{\prime \prime}\|b\|_{\infty} \sup _{y \in G_{\theta}, \zeta \in m\left(\mathbb{C}^{n}\right)}\left\{|\zeta|^{-n}\left|\widehat{\psi}_{1 /|\zeta|}(\zeta /|\zeta|-y /|\zeta|)\right|\right\} \\
& \times \int_{\mathbb{R}^{n}} e^{-t\langle x, m\rangle|\xi|} d \xi \\
\leq & \frac{C_{\nu}^{\prime \prime \prime}\|b\|_{\infty}}{t^{n}\langle x, m\rangle^{n}} \sup _{y \in G_{\theta}, \zeta \in m\left(\mathbb{C}^{n}\right)}\left\{|\zeta|^{-n}\left|\widehat{\psi}_{1 /|\zeta|}(\zeta /|\zeta|-y /|\zeta|)\right|\right\}
\end{aligned}
$$

Hence we obtain decay as $t|x| \rightarrow \infty$ in $S_{\nu^{\prime}}^{\circ}\left(\mathbb{R}^{n+1}\right)$. To see this, let $\nu^{\prime \prime}$ satisfy $\nu^{\prime}<\nu^{\prime \prime}<\theta$. Then for all $x \in S_{\nu^{\prime}}^{\circ}\left(\mathbb{R}^{n+1}\right)$ we can find a unit vector $m=$ $m_{0} e_{0}+\boldsymbol{m} \in \mathbb{R}^{n+1}$ satisfying $m_{0}>|\boldsymbol{m}| \cot \nu^{\prime \prime}$ such that $\langle x, m\rangle>a|x|$, where $a$ depends only on $\nu^{\prime}$ and $\nu^{\prime \prime}$.

We now estimate the convergence of $\left(b \cdot \ell \psi_{t}^{2}\right)_{+}(x)$ as $t x \rightarrow 0$ in $S_{\nu^{\prime}}^{\circ}\left(\mathbb{R}^{n+1}\right)$. Set

$$
V_{\theta}(\zeta)=\sup _{y \in G_{\theta}}\left|\widehat{\psi}_{1 /|\zeta|}(\zeta /|\zeta|-y /|\zeta|)\right|, \quad \zeta \in S_{\nu^{\prime}}\left(\mathbb{C}^{n}\right)
$$

Then as shown above, $V_{\theta}(\zeta)=O\left(|\zeta|^{-1}\right)$ as $|\zeta| \rightarrow \infty$ for $\zeta \in S_{\nu^{\prime}}\left(\mathbb{C}^{n}\right)$, and $V_{\theta}(\zeta)=O\left(|\zeta|^{n}\right)$ as $|\zeta| \rightarrow 0$ for $\zeta \in S_{\nu^{\prime}}\left(\mathbb{C}^{n}\right)$.

Because

$$
\left(b \cdot \ell \psi_{t}^{2}\right)_{+}(0)=\frac{1}{(2 \pi)^{n}} \int_{m\left(\mathbb{C}^{n}\right)} \chi_{+}(\zeta)\left(b \cdot \psi_{t}^{2}\right)^{\wedge}(\zeta) d \zeta_{1} \wedge \cdots \wedge d \zeta_{n},
$$

the number $\left|\left(b \cdot \ell \psi_{t}^{2}\right)_{+}(x)-\left(b \cdot \ell \psi_{t}^{2}\right)_{+}(0)\right|$ is estimated by

$$
C_{\nu}^{\prime \prime}\|b\|_{\infty} \int_{m\left(\mathbb{C}^{n}\right)}\left|e_{+}(t x, \zeta)-\chi_{+}(\zeta)\right| V_{\theta}(\zeta) \frac{\left|d \zeta_{1} \wedge \cdots \wedge d \zeta_{n}\right|}{|\zeta|^{n}} .
$$


For notational simplicity, replace $t x$ by $x$. Then for $|x| \leq 1$, we have

$$
\begin{aligned}
& \int_{m\left(\mathbb{C}^{n}\right) \cap\left\{|\zeta| \geq|x|^{-1 / 2}\right\}}\left|e_{+}(x, \zeta)-\chi_{+}(\zeta)\right| V_{\theta}(\zeta) \frac{\left|d \zeta_{1} \wedge \cdots \wedge d \zeta_{n}\right|}{|\zeta|^{n}} \\
\leq & \int_{m\left(\mathbb{C}^{n}\right) \cap\left\{|\zeta| \geq|x|^{-1 / 2}\right\}} V_{\theta}(\zeta) \frac{\left|d \zeta_{1} \wedge \cdots \wedge d \zeta_{n}\right|}{|\zeta|^{n}} \\
\leq & C^{\prime} \int_{m\left(\mathbb{C}^{n}\right) \cap\left\{|\zeta| \geq|x|^{-1 / 2}\right\}} \frac{\left|d \zeta_{1} \wedge \cdots \wedge d \zeta_{n}\right|}{|\zeta|^{n+1}} \leq C^{\prime \prime} \int_{|x|^{-1 / 2}}^{\infty} \frac{d r}{r^{2}}=C^{\prime \prime \prime}|x|^{1 / 2} .
\end{aligned}
$$

On the other hand,

$$
\begin{aligned}
& \int_{m\left(\mathbb{C}^{n}\right) \cap\left\{|\zeta| \leq|x|^{-1 / 2}\right\}}\left|e_{+}(x, \zeta)-\chi_{+}(\zeta)\right| V_{\theta}(\zeta) \frac{\left|d \zeta_{1} \wedge \cdots \wedge d \zeta_{n}\right|}{|\zeta|^{n}} \\
& \quad \leq|x|^{1 / 2} \sup _{|\zeta| \leq|x|^{-1 / 2}, \zeta \in m\left(\mathbb{C}^{n}\right)} \frac{\left|e_{+}(x, \zeta)-\chi_{+}(\zeta)\right|}{|x|^{1 / 2}} \int_{m\left(\mathbb{C}^{n}\right)} V_{\theta}(\zeta) \frac{\left|d \zeta_{1} \wedge \cdots \wedge d \zeta_{n}\right|}{|\zeta|^{n}} .
\end{aligned}
$$

Because $\langle x, m\rangle$ is comparable to $|x|$ for $x \in S_{\nu^{\prime}}^{\circ}\left(\mathbb{R}^{n+1}\right)$ and $|\zeta|$ is comparable to $\Re|\zeta|_{\mathbb{C}}$ for $\zeta \in m\left(\mathbb{C}^{n}\right)$, there exist $a>0$ and $C>0$ such that

$$
\begin{aligned}
\sup _{|\zeta| \leq|x|^{-1 / 2}, \zeta \in m\left(\mathbb{C}^{n}\right)} \frac{\left|e_{+}(x, \zeta)-\chi_{+}(\zeta)\right|}{|x|^{1 / 2}} & \leq C \sup _{|\zeta| \leq|x|^{-1 / 2}, \zeta \in m\left(\mathbb{C}^{n}\right)} \frac{1-e^{-a|x||\zeta|}}{|x|^{1 / 2}} \\
& =C \frac{1-e^{-a|x|^{1 / 2}}}{|x|^{1 / 2}} \leq a C .
\end{aligned}
$$

It follows that $\left|\left(b \cdot \ell \psi_{t}^{2}\right)_{+}(x)-\left(b \cdot \ell \psi_{t}^{2}\right)_{+}(0)\right|$ is $O\left((t|x|)^{1 / 2}\right)$ as $t|x| \rightarrow 0$ in $S_{\nu^{\prime}}\left(\mathbb{R}^{n+1}\right)$.

If in the integral representation (33), $x \in \mathbb{R}^{n}$ and $m_{0} \rightarrow e_{0}, m_{0}^{\prime} \rightarrow e_{0}$, then we obtain

$$
\begin{aligned}
b \cdot \ell \psi_{t}^{2}(x)= & \frac{1}{(2 \pi)^{n}} \int_{\mathbb{R}^{n}} e^{i\langle x, \xi\rangle} \chi_{+}(\xi)\left(b \cdot \psi_{t}^{2}\right)^{\wedge}(\xi) d \xi \\
& +\frac{1}{(2 \pi)^{n}} \int_{\mathbb{R}^{n}} e^{i\langle x, \xi\rangle} \chi_{-}(\xi)\left(b \cdot \psi_{t}^{2}\right)^{\wedge}(\xi) d \xi=\left(b \cdot \psi_{t}^{2}\right)(x),
\end{aligned}
$$

as expected, because $\chi_{+}(\xi)+\chi_{-}(\xi)=1$ for all $\xi \in \mathbb{R}^{n} \backslash\{0\}$.

Now write the second integral in $(33)$ as $\left(b \cdot \ell \psi_{t}^{2}\right)_{-}$. Then

$$
\left(b \cdot \ell \psi_{t}^{2}\right)_{+}(0)+\left(b \cdot \ell \psi_{t}^{2}\right)_{-}(0)=\left(b \cdot \psi_{t}^{2}\right)(0)=0,
$$

and as above, we see $\left|\left(b \cdot \ell \psi_{t}^{2}\right)_{-}(x)-\left(b \cdot \ell \psi_{t}^{2}\right)_{-}(0)\right|$ is $O\left((t|x|)^{1 / 2}\right)$ as $t|x| \rightarrow 0$ in $S_{\nu^{\prime}}\left(\mathbb{R}^{n+1}\right)$ and $\left|\left(b \cdot \ell \psi_{t}^{2}\right)_{-}(x)\right|$ is $O\left((t|x|)^{-n}\right)$ as $t|x| \rightarrow \infty$ in $S_{\nu^{\prime}}\left(\mathbb{R}^{n+1}\right)$. 
Because $\left(b \cdot \ell \psi_{t}^{2}\right)(x)=\left(b \cdot \ell \psi_{t}^{2}\right)_{+}(x)+\left(b \cdot \ell \psi_{t}^{2}\right)_{-}(x)$ for all $x \in S_{\nu^{\prime}}\left(\mathbb{R}^{n+1}\right)$, it follows that $\left|\left(b \cdot \ell \psi_{t}^{2}\right)(x)\right|$ is $O\left((t|x|)^{1 / 2}\right)$ as $t|x| \rightarrow 0$ in $S_{\nu^{\prime}}\left(\mathbb{R}^{n+1}\right)$ and $\left|\left(b \cdot \ell \psi_{t}^{2}\right)(x)\right|$ is $O\left((t|x|)^{-n}\right)$ as $t|x| \rightarrow \infty$ in $S_{\nu^{\prime}}\left(\mathbb{R}^{n+1}\right)$. All constants we have calculated are proportional to the supremum norm $\|b\|_{\infty}$ of $b$ on $S_{\nu}\left(\mathbb{C}^{n}\right)$, so the convergence is uniform for $\|b\|_{\infty} \leq 1$.

Because $b \cdot \ell \psi_{t}^{2}$ has decay $O(t|x|)$ as $t|x| \rightarrow 0$ in $\mathbb{R}^{n}$ and $b \cdot \ell \psi_{t}^{2}$ has exponential decay as $t|x| \rightarrow \infty$ in $\mathbb{R}^{n}$, the estimate (32) may not be the best possible.

Proof of Theorem 2.4. Let $0<\nu<\pi / 2$. It remains to show that if $b: S_{\nu}^{\circ}\left(\mathbb{C}^{n}\right) \rightarrow C \ell\left(\mathbb{C}^{n}\right)$ is a uniformly bounded holomorphic function, then there exists a left monogenic function $f: S_{\nu}^{\circ}\left(\mathbb{R}^{n+1}\right) \rightarrow C \ell\left(\mathbb{C}^{n}\right)$, uniformly bounded on subsectors of $S_{\nu}^{\circ}\left(\mathbb{R}^{n+1}\right)$, such that $b=\widetilde{f}$ is represented by the Cauchy integral formula (13). Then $f$ is the left monogenic extension to $S_{\nu}^{\circ}\left(\mathbb{R}^{n+1}\right)$ of the restriction of $b$ to $\mathbb{R}^{n} \backslash\{0\}$.

For each $\zeta \in \mathbb{C}^{n}$ such that $|\zeta|_{\mathbb{C}}^{2} \notin(-\infty, 0]$, set $\phi(\zeta)=\chi_{-}(\zeta)|\zeta|_{\mathbb{C}} e^{-|\zeta|_{\mathbb{C}}}$ and $\phi_{t}(\zeta)=\phi(t \zeta)$ for $t>0$. A similar argument to the proof of Lemma 4.5 shows that we may substitute $\phi$ for $\psi$ and the same statement holds. The bound (27) now holds for $x \in C_{\nu^{\prime}}^{+}$when $\phi$ is substituted for $\psi$, because in formula $(28)$ for $\left(b \cdot \phi_{t}\right)^{\wedge}$, the expression $e_{+}(-x, \zeta)$ is replaced by $e_{-}(-x, \zeta)$ for all $\langle x, m\rangle>0$ and $\zeta \in m\left(\mathbb{C}^{n}\right)$. Set

$$
f(x)=4 \int_{0}^{\infty}\left(\left(b \cdot \ell \psi_{t}^{2}\right)(x)+\left(b \cdot \ell \phi_{t}^{2}\right)(x)\right) \frac{d t}{t} .
$$

Then according to Lemma 4.5 and its analogue when $\psi$ is replaced by $\phi$, the decay estimates (32) ensure that the integral converges absolutely for all $x \in S_{\nu}^{\circ}\left(\mathbb{R}^{n+1}\right)$ and $f$ is a left monogenic function in $S_{\nu}^{\circ}\left(\mathbb{R}^{n+1}\right)$, because $b \cdot \ell \psi_{t}^{2}$ and $b \cdot \ell \phi_{t}^{2}$ are both left monogenic functions there. If $x \in \mathbb{R}^{n} \backslash\{0\}$, then we have

$$
\begin{aligned}
f(x) & =4 b(x) \int_{0}^{\infty}\left(\psi_{t}^{2}(x)+\phi_{t}^{2}(x)\right) \frac{d t}{t} \\
& =4 b(x) \int_{0}^{\infty}\left(\chi_{+}(x)+\chi_{-}(x)\right)(t|x|)^{2} e^{-2 t|x|} \frac{d t}{t}=4 b(x) \int_{0}^{\infty} t e^{-2 t} d t=b(x) .
\end{aligned}
$$

Here we have used the facts that $\chi_{ \pm}(x)$ are projections and $\chi_{+}(x)+\chi_{-}(x)=1$ for each nonzero $x \in \mathbb{R}^{n}$. The uniformity of the decay estimates (32) for $\|b\|_{\infty} \leq 1$ ensures that for every $0<\nu^{\prime}<\nu$, there exists $C_{\nu^{\prime}}>0$ independent of $b$ such that the bound $|f(x)| \leq C_{\nu^{\prime}}\|b\|_{\infty}$ holds for all $x \in S_{\nu^{\prime}}^{\circ}\left(\mathbb{R}^{n+1}\right)$. This completes the proof of Theorem 2.4.

REMARK 4.6. Formula (39) represents the inverse mapping of $f \mapsto \widetilde{f}$ defined by the Cauchy integral formula (13) with $b=\widetilde{f}$. Another way of 
looking at (39) is to set

$$
\Psi(z)=2\left(\chi_{\Re(z)>0} z e^{-z}+\chi_{\Re(z)<0} z e^{z}\right), \quad z \in S_{\nu}^{\circ}(\mathbb{C}) .
$$

Then $\int_{0}^{\infty} \Psi^{2}(t) t^{-1} d t=\int_{0}^{\infty} \Psi^{2}(-t) t^{-1} d t=1$. As noted in Section 2 , the spectral projections $\chi_{ \pm}(\zeta)$ are associated with multiplication by $i \zeta$ on the Clifford algebra $C \ell\left(\mathbb{C}^{n}\right)$. Let $\widetilde{\Psi}$ be the function of $\zeta \in \mathbb{C}^{n}$ defined by the functional calculus

$$
\widetilde{\Psi}(\zeta)=\Psi\{i \zeta\}=\Psi\left(|\zeta|_{\mathbb{C}}\right) \chi_{+}(\zeta)+\Psi(-|\zeta| \mathbb{C}) \chi_{-}(\zeta)
$$

for multiplication by $i \zeta$ and set $\widetilde{\Psi}_{t}(\zeta)=\widetilde{\Psi}(t \zeta)$ for $t>0$. Then formula (39) may be written as

$$
f=\int_{0}^{\infty} b \cdot \ell \widetilde{\Psi}_{t}^{2} \frac{d t}{t}
$$

We have shown that by multiplying a bounded holomorphic function $b$ in a sector by a suitable holomorphic function $\widetilde{\Psi}_{t}$ with decay at zero and infinity, the product $b \cdot \widetilde{\Psi}_{t}^{2}$ may be extended from $\mathbb{R}^{n} \backslash\{0\}$ to a left monogenic function $b \cdot \ell \widetilde{\Psi}_{t}^{2}$ for each $t>0$ (cf. [10, p. 65]). Then we can integrate out the scaling factor $t$. It is plausible that similar techniques could be applied to domains other than sectors and other Hardy spaces of functions by using decompositions of functions other than in terms of trigonometric functions.

5. Application to operator theory. Suppose that $T: \mathcal{D}(T) \rightarrow \mathcal{H}$ is a single densely defined linear operator acting in the Hilbert space $\mathcal{H}$. If $0 \leq \omega<\pi / 2$, then $T$ is said to be of type $\omega$ if $\sigma(T) \subset S_{\omega}(\mathbb{C})$ and for each $\nu>\omega$, there exists $C_{\nu}>0$ such that

$$
\left\|(z I-T)^{-1}\right\| \leq C_{\nu}|z|^{-1}, \quad z \notin S_{\nu}(\mathbb{C}) .
$$

Then the bounded linear operator $f(T)$ is defined by formula (1) for any function $f$ satisfying the bounds $(47)$ in $S_{\nu}(\mathbb{C})$ in the case $n=1$ and $A=T$. The contour $C$ can be taken to be $\{z \in \mathbb{C}:|\Im(z)|=|\Re(z)| \tan \theta\}$, with $\omega<\theta<\nu$.

The operator $T$ of type $\omega$ is said to have a bounded $H^{\infty}$-functional calculus if for each $\omega<\nu<\pi / 2$, there exists an algebra homomorphism $f \mapsto f(T)$ from $H^{\infty}\left(S_{\nu}^{\circ}(\mathbb{C})\right)$ to $\mathcal{L}(\mathcal{H})$ agreeing with $(1)$ and a positive number $C_{\nu}$ such that $\|f(T)\| \leq C_{\nu}\|f\|_{\infty}$ for all $f \in H^{\infty}\left(S_{\nu}^{\circ}(\mathbb{C})\right)$.

The following result is from [7, Theorem 6.2.2].

TheOREM 5.1. Suppose that $T$ is a one-to-one operator of type $\omega$ in $\mathcal{H}$. Then $T$ has a bounded $H^{\infty}$-functional calculus if and only if for every $\omega<$ $\nu<\pi / 2$, there exists $c_{\nu}>0$ such that $T$ and its adjoint $T^{*}$ satisfy the square 
function estimates

$$
\begin{aligned}
& \int_{0}^{\infty}\left\|\psi_{t}(T) u\right\|^{2} \frac{d t}{t} \leq c_{\nu}\|u\|^{2}, \quad u \in \mathcal{H}, \\
& \int_{0}^{\infty}\left\|\psi_{t}\left(T^{*}\right) u\right\|^{2} \frac{d t}{t} \leq c_{\nu}\|u\|^{2}, \quad u \in \mathcal{H},
\end{aligned}
$$

for some function (every function) $\psi \in H^{\infty}\left(S_{\nu}^{\circ}(\mathbb{C})\right)$ which satisfies

$$
\begin{aligned}
& \int_{0}^{\infty} \psi^{3}(t) \frac{d t}{t}=\int_{0}^{\infty} \psi^{3}(-t) \frac{d t}{t}=1, \\
& |\psi(z)| \leq C_{\nu} \frac{|z|^{s}}{1+|z|^{2 s}}, \quad z \in S_{\nu}^{\circ}(\mathbb{C}),
\end{aligned}
$$

for some $s>0$. Here $\psi_{t}(z)=\psi(t z)$ for $z \in S_{\nu}^{\circ}(\mathbb{C})$.

We now use formula (2) to generalise this result to $n$-tuples of commuting operators acting in a Hilbert space $\mathcal{H}$.

The $(n-1)$-sphere in $\mathbb{R}^{n}$ is denoted by $S^{n-1}$. The set of $s \in S^{n-1}$ with nonzero coordinates $s_{j}$ for every $j=1, \ldots, n$ is denoted by $S_{0}^{n-1}$. Then $S_{0}^{n-1}$ is a dense open subset of $S^{n-1}$ with full surface measure.

Definition 5.2. Let $X$ be a Banach space and let $A=\left(A_{1}, \ldots, A_{n}\right)$ be an $n$-tuple of densely defined linear operators $A_{j}: \mathcal{D}\left(A_{j}\right) \rightarrow X$ acting in $X$ such that $\bigcap_{j=1}^{n} \mathcal{D}\left(A_{j}\right)$ is dense in $X$ and let $0 \leq \omega<\pi / 2$. Then $A$ is said to be uniformly of type $\omega$ if for all $s \in S_{0}^{n-1}$, the densely defined operator $\langle A, s\rangle:=\sum_{j=1}^{n} A_{j} s_{j}$ is closed, $\sigma(\langle A, s\rangle) \subset S_{\omega}(\mathbb{C})$ and for each $\nu>\omega$, there exists $C_{\nu}>0$ such that

$$
\left\|(z I-\langle A, s\rangle)^{-1}\right\| \leq C_{\nu}|z|^{-1}, \quad z \notin S_{\nu}(\mathbb{C}), s \in S_{0}^{n-1} .
$$

It follows that $s \mapsto\langle A, s\rangle$ is continuous on $S_{0}^{n-1}$ in the sense of strong resolvent convergence [6, Theorem VIII.1.5].

If $A$ is uniformly of type $\omega$, it turns out that we can define the Cauchy kernel $G_{x}(A)$ for the $n$-tuple $A$ by the plane wave formula

$$
\begin{aligned}
G_{x}(A)= & \frac{(n-1) !}{2}\left(\frac{i}{2 \pi}\right)^{n} \operatorname{sgn}\left(x_{0}\right)^{n-1} \\
& \times \int_{S^{n-1}}\left(e_{0}+i s\right)\left(\langle\boldsymbol{x}, s\rangle I-\langle A, s\rangle-x_{0} s I\right)^{-n} d s
\end{aligned}
$$

for all $x=x_{0} e_{0}+\boldsymbol{x}$ with $x_{0}$ a nonzero real number and $\boldsymbol{x} \in \mathbb{R}^{n}$. Here $S^{n-1}$ is the unit $(n-1)$-sphere in $\mathbb{R}^{n}$, ds is surface measure and the inverse power $\left(\langle\boldsymbol{x} I-A, s\rangle-x_{0} s\right)^{-n}$ is taken in the Clifford module $\mathcal{L}(X) \otimes C \ell\left(\mathbb{C}^{n}\right)$, which is identified with the set $\mathcal{L}_{(n)}\left(X_{(n)}\right)$ of all left module homomorphisms of $X_{(n)}=X \otimes C \ell\left(\mathbb{C}^{n}\right)$. 
Suppose that $\omega<\nu<\pi / 2$ and $f$ is a left monogenic function defined on $S_{\nu}^{\circ}\left(\mathbb{R}^{n+1}\right)$ such that for every $0<\theta<\nu$ there exist $C_{\theta}>0$ and $s>0$ such that

$$
|f(x)| \leq C_{\theta} \frac{|x|^{s}}{1+|x|^{2 s}}, \quad x \in S_{\theta}^{\circ}\left(\mathbb{R}^{n+1}\right) .
$$

Now if $\omega<\theta<\nu$ and

$$
H_{\theta}=\left\{x \in \mathbb{R}^{n+1}: x=x_{0} e_{0}+\boldsymbol{x},\left|x_{0}\right| /|x|=\tan \theta\right\} \subset S_{\nu}^{\circ}\left(\mathbb{R}^{n+1}\right),
$$

then it is easy to check that $x \mapsto G_{x}(A) \boldsymbol{n}(x) f(x)$ is integrable with respect to $n$-dimensional surface measure $\mu$ on $H_{\theta}$. Therefore, we define

$$
f(A)=\int_{H_{\theta}} G_{x}(A) \boldsymbol{n}(x) f(x) d \mu(x) .
$$

If $\psi: \mathbb{R}^{n+1} \backslash\{0\} \rightarrow C \ell\left(\mathbb{C}^{n}\right)$ has a left monogenic extension $\widetilde{\psi}$ to $S_{\nu}^{\circ}\left(\mathbb{R}^{n+1}\right)$ that satisfies the bound (47) for all $0<\theta<\nu$, then $\widetilde{\psi}(A)$ is written just as $\psi(A)$. The hypersurface $H_{\theta}$ can be varied in the region where $x \mapsto G_{x}(A)$ is two-sided monogenic in the Clifford module $\mathcal{L}(X) \otimes C \ell\left(\mathbb{C}^{n}\right)$ and $f$ is left monogenic in $S_{\nu}^{\circ}\left(\mathbb{R}^{n+1}\right)$.

Theorem 5.3. Let $A=\left(A_{1}, \ldots, A_{n}\right)$ be an $n$-tuple of densely defined commuting linear operators $A_{j}: \mathcal{D}\left(A_{j}\right) \rightarrow \mathcal{H}$ acting in a Hilbert space $\mathcal{H}$ such that $\bigcap_{j=1}^{n} \mathcal{D}\left(A_{j}\right)$ is dense in $\mathcal{H}$. Suppose that $0 \leq \omega<\pi / 2$ and $A$ is uniformly of type $\omega$. If $T=i\left(A_{1} e_{1}+\cdots+A_{n} e_{n}\right)$ is a one-to-one operator of type $\omega$ acting in $\mathcal{H}_{(n)}=\mathcal{H} \otimes C \ell\left(\mathbb{C}^{n}\right)$ and $T$ has an $H^{\infty}$-functional calculus, then the $n$-tuple $A$ has a bounded $H^{\infty}$-functional calculus on $S_{\nu}^{\circ}\left(\mathbb{C}^{n}\right)$ for any $\omega<\nu<\pi / 2$, that is, there exists a homomorphism $b \mapsto b(A), b \in$ $H^{\infty}\left(S_{\nu}^{\circ}\left(\mathbb{C}^{n}\right)\right)$, from $H^{\infty}\left(S_{\nu}^{\circ}\left(\mathbb{C}^{n}\right)\right)$ into $\mathcal{L}_{(n)}\left(\mathcal{H}_{(n)}\right)$ and there exists $C_{\nu}>0$ such that $\|b(A)\| \leq C_{\nu}\|b\|_{\infty}$ for all $b \in H^{\infty}\left(S_{\nu}^{\circ}\left(\mathbb{C}^{n}\right)\right)$.

Moreover, if $f$ is the unique two-sided monogenic function defined on $S_{\nu}^{\circ}\left(\mathbb{R}^{n+1}\right)$ such that $b=\widetilde{f}$, as in Theorem 2.4 , and $f$ satisfies the bound $(47)$, then $b(A)=f(A)$ is given by formula (49).

Proof. By assumption, the operator $T$ has an $H^{\infty}$-functional calculus, so there exists $\psi \in H^{\infty}\left(S_{\nu}^{\circ}(\mathbb{C})\right)$ satisfying (41)-(44). In fact, we are at liberty to choose the function $\psi$, so we set $\psi(z)=C\left(\chi_{\Re(z)>0} z e^{-z}+\chi_{\Re(z)<0} z e^{z}\right)$ for all $z \in S_{\nu}^{\circ}(\mathbb{C})$. We choose the constant $C$ later.

Following [7, Theorem 6.4.3], our aim is to define $b(A)$ for $b \in H^{\infty}\left(S_{\nu}^{\circ}\left(\mathbb{C}^{n}\right)\right)$ by the formula

$$
(b(A) u, v)=\int_{0}^{\infty}\left(\left(b \phi_{t}\right)(A) \psi_{t}(T) u, \psi_{t}(T)^{*} v\right) \frac{d t}{t}
$$

for all $u, v \in \mathcal{H}_{(n)}$. The function $\phi: S_{\nu}^{\circ}\left(\mathbb{C}^{n}\right) \rightarrow \mathbb{C}$ is constructed from $\psi$ by 
setting

$$
\phi(\zeta)=\psi^{2}\{i \zeta\}=\psi^{2}\left(|\zeta|_{\mathbb{C}}\right) \chi_{+}(\zeta)+\psi^{2}\left(-|\zeta|_{\mathbb{C}}\right) \chi_{-}(\zeta)
$$

for all $\zeta \in S_{\nu}^{\circ}\left(\mathbb{C}^{n}\right)$, by the functional calculus for multiplication by $i \zeta$.

According to Corollary 2.5, the mapping $f \mapsto \widetilde{f}$ given by the Cauchy integral formula (13) is an isomorphism of the Fréchet algebras $H_{\ell}^{\infty}\left(S_{\nu}^{\circ}\left(\mathbb{R}^{n+1}\right)\right)$ and $H^{\infty}\left(S_{\nu}^{\circ}\left(\mathbb{C}^{n}\right)\right)$, so there is a left monogenic function $b \cdot \ell \phi_{t} \in H_{\ell}^{\infty}\left(S_{\nu}^{\circ}\left(\mathbb{R}^{n+1}\right)\right)$ corresponding to the product function $\zeta \mapsto b(\zeta) \phi_{t}(\zeta), \zeta \in S_{\nu}^{\circ}\left(\mathbb{C}^{n}\right)$, such that $b \cdot \phi_{t}=\left(b \cdot \ell \phi_{t}\right)^{\sim}$.

We know from the estimates (32) that $b \cdot \ell \phi_{t}$ satisfies the bound (47) with $s=1 / 2$ and $C_{\theta}$ proportional to $\|b\|_{\infty}$, so $\left(b \phi_{t}\right)(A)=b \cdot \ell \phi_{t}(A)$ is defined by formula (49) and we have

$$
\begin{aligned}
& \int_{0}^{\infty}\left|\left(\left(b \phi_{t}\right)(A) \psi_{t}(i T) u, \psi_{t}(i T)^{*} v\right)\right| \frac{d t}{t} \\
& \quad \leq \sup _{t>0}\left\|\left(b \phi_{t}\right)(A)\right\|\left\{\int_{0}^{\infty}\left\|\psi_{t}(i T) u\right\|^{2} \frac{d t}{t}\right\}^{1 / 2}\left\{\int_{0}^{\infty}\left\|\psi_{t}(i T)^{*} u\right\|^{2} \frac{d t}{t}\right\}^{1 / 2} \\
& \quad \leq C^{\prime}\|b\|_{\infty}\|u\|\|v\| .
\end{aligned}
$$

If we choose $C>0$ such that $\int_{0}^{\infty} \psi^{4}(t) d t / t=1$, then we obtain the desired functional calculus along the lines of [7, Theorem 6.4.3].

The assumptions of Theorem 5.3 are satisfied if the $n$-tuple $A$ consists of differentiation operators on a Lipschitz surface [8].

\section{References}

[1] R. F. V. Anderson, The Weyl functional calculus, J. Funct. Anal. 4 (1969), 240-267.

[2] F. Brackx, R. Delanghe and F. Sommen, Clifford Analysis, Res. Notes in Math. 76, Pitman, Boston, MA, 1982.

[3] R. Delanghe, F. Sommen and V. Souček, Clifford Algebras and Spinor-Valued Functions. A Function Theory for the Dirac Operator, Kluwer, Dordrecht, 1992.

[4] B. Jefferies and A. McIntosh, The Weyl calculus and Clifford analysis, Bull. Austral. Math. Soc. 57 (1998), 329-341.

[5] B. Jefferies, A. McIntosh and J. Picton-Warlow, The monogenic functional calculus, Studia Math. 136 (1999), 99-119.

[6] T. Kato, Perturbation Theory for Linear Operators, 2nd ed., Springer, Berlin, 1980.

[7] C. Li and A. McIntosh, Clifford algebras and $H_{\infty}$ functional calculi of commuting operators, in: Clifford Algebras in Analysis and Related Topics (Fayetteville, AR, 1993), Stud. Adv. Math., CRC, Boca Raton, FL, 1996, 89-101.

[8] C. Li, A. McIntosh and T. Qian, Clifford algebras, Fourier transforms and singular convolution operators on Lipschitz surfaces, Rev. Mat. Iberoamericana 10 (1994), $665-721$.

[9] A. McIntosh, Operators which have an $H_{\infty}$ functional calculus, in: Miniconference on Operator Theory and Partial Differential Equations (North Ryde, 1986), Proc. 
Centre Math. Anal. Austral. Nat. Univ. 14, Austral. Nat. Univ., Canberra, 1986, 210-231.

[10] A. McIntosh, Clifford algebras, Fourier theory, singular integrals, and harmonic functions on Lipschitz domains, in: Clifford Algebras in Analysis and Related Topics (Fayetteville, AR, 1993), Stud. Adv. Math., CRC, Boca Raton, FL, 1996, 33-87.

[11] M. Mitrea, Clifford Wavelets, Singular Integrals and Hardy Spaces, Springer, Berlin, 1994.

School of Mathematics

The University of New South Wales

Sydney, NSW 2052, Australia

E-mail: b.jefferies@unsw.edu.au

Received March 10, 2003

Revised version November 12, 2003 